\title{
FREDHOLM, HODGE AND LIOUVILLE THEOREMS ON NONCOMPACT MANIFOLDS
}

\begin{abstract}
ROBERT LOCKHART
Abstract. Fredholm, Liouville, Hodge, and $L^{2}$-cohomology theorems are proved for Laplacians associated with a class of metrics defined on manifolds that have finitely many ends. The metrics are conformal to ones that are asymptotically translation invariant. They are not necessarily complete. The Fredholm results are, of necessity, with respect to weighted Sobolev spaces. Embedding and compact embedding theorems are also proved for these spaces.
\end{abstract}

Two of the most useful facts in analysis on a compact Riemannian manifold are that the Laplacian is Fredholm and its kernel consists of closed and coclosed forms that provide unique representatives for all the de Rham cohomology classes. Naturally one would like to extend these results to noncompact manifolds. The first such result in this direction is due to Kodaira [9, 12, p. 165]. It is that $L^{2}\left(\bigwedge^{q} M, g\right)$ is the orthogonal direct sum of $\overline{d C_{0}^{\infty}\left(\wedge^{q-1} M\right)}, \overline{d_{g}^{*} C_{0}^{\infty}\left(\bigwedge^{q+1} M\right)}$, and

$$
\tilde{\mathscr{S}}^{2}\left(\bigwedge^{q} M, g\right)=\left\{\sigma \in L^{2}\left(\bigwedge^{q} M, g\right) \mid d \sigma=d_{g}^{*} \sigma=0\right\} .
$$

Unfortunately, if no restriction is made on the manifold or the metric, one cannot improve on this. The Laplacian need not be Fredholm; $L^{2}$-harmonic forms need not be closed or coclosed; even if they are closed and coclosed, the space of harmonic $L^{2}$ forms need not be finite dimensional (see [7]); and even if the Laplacian is Fredholm and $L^{2}$-harmonic forms are closed and coclosed, those forms need not provide unique or total representation of de Rham cohomology.

Thus one of the main questions in analysis on noncompact manifolds is what conditions on $M$ and $g$ allow one to carry over the Fredholm and Hodge theorems for compact manifolds and, if they cannot be carried over completely, to what extent can they be? In the case of Hodge's theorem, i.e., properties of the space of $L^{2}$ harmonic forms, this question has been quite actively investigated recently (see [2-8, 11, and 13]).

For instance, one of the results of Atiyah, Patodi, and Singer in [2] is that if a manifold has cylindrical ends then $\tilde{\mathfrak{S}}^{2}\left(\wedge^{g} M, g\right)$ is naturally isomorphic to the image of $H_{\text {comp }}^{q}(M)$ in $H_{D R}^{q}(M)$ (see [2, Proposition 4.9]). In [11], Müller investigates the spectrum of the Laplacian on manifolds that outside a compact set are $\Omega \times \mathbf{R}^{+}$, with

Received by the editors August 30, 1985 and, in revised form, April 10, 1986.

1980 Mathematics Subject Classification (1985 Revision). Primary 58G10, 35J05.

(c)1987 American Mathematical Society $0002-9947 / 87 \$ 1.00+\$ .25$ per page 
$\Omega$ compact, and are equipped with a metric that on $\Omega \times[1, \infty)$ is of the form $z^{-2}\left(d z^{2}+d s_{\Omega}^{2}\right)$ with $d s_{\Omega}^{2}$ a metric on $\Omega$. A corollary of his Proposition 6.9 is that $\tilde{\mathfrak{S}}^{2}\left(\wedge^{q} M, g\right)$ is finite dimensional. In [4] Cheeger investigates, among other things, the $L^{2}$ cohomology of spaces of the form $M=\Omega \times(0,1)$ equipped with the metric $g=d r^{2}+r^{2 \alpha} d s_{\Omega}^{2}$ with $\alpha \geqslant 1$. One of the consequences of Lemma 3.4 of [4] is that if $\Omega$ is compact then $\operatorname{dim} \tilde{\mathfrak{S}}^{2}\left(\wedge^{q} M, g\right) \leqslant \operatorname{dim} H_{D R}^{q}(M)$. Using a separation of variables argument like that in [3] one can show that in fact $\operatorname{dim} \tilde{\mathscr{S}}^{2}\left(\bigwedge^{q} M, g\right)=\operatorname{dim} H_{D R}^{q}(M)$ when $2 q<\operatorname{dim} M$.

In this paper we also examine the properties of $L^{2}$ harmonic forms on a class of manifolds equipped with certain metrics. However, unlike previous investigators who by and large treated $\Delta, d$, and $\delta$ as unbounded operators on $L^{2}$, we consider the Fredholm properties of the Laplacian with respect to a particular family of weighted Sobolev spaces. There are three reasons for this. First of all, it allows for the consideration of a larger class of metrics. Secondly, a Fredholm theory for the Laplacian is as important to analysts as a Hodge theory. In particular, such a theory should be of use in nonlinear analysis on noncompact manifolds. It is for this reason that we do not restrict attention to weighted $L^{2}$ Sobolev spaces, but rather work with weighted $L^{p}$ spaces for $1<p<\infty$. It is also for this reason that we present several embedding theorems for the Sobolev spaces which we use. Thirdly, by using weights, we are able to prove Liouville type theorems for harmonic forms.

To be more precise, the noncompact manifolds we consider have finitely many ends. Thus $M$ contains a compact manifold, $M_{0}$, with boundary; $\partial M_{0}$ has finitely many components, and $M_{\infty}=M-M_{0}=\partial M_{0} \times \mathbf{R}^{+}$. On such a manifold there is a natural $\mathbf{R}^{+}$-action on each end and so the notion of asymptotically translation invariant metric makes sense (see $\S 2$ ). If $h$ is such a metric, then $g=e^{2 \rho} h$ is said to be admissible if all the covariant derivatives of $\rho$ have a limit at infinity (see 2.3.1).

Examples of admissible metrics abound. For instance, every metric that is asymptotically translation invariant is admissible. For $h$ such a metric, the metric that on $M_{\infty}$ is $g=e^{2 z} h$ is admissible. As a special case of this, suppose $h=d z^{2}+$ $d s_{\partial M_{0}}^{2}$ on $M_{\infty}$. Letting $r=e^{z}$ we see that $g=d r^{2}+r^{2} d s_{\partial M_{0}}^{2}$ on $(\omega, r) \in \partial M_{0} \times$ $(1, \infty)=M_{\infty}$. In particular if $\partial M_{0}$ is $k$-copies of $S^{n-1}$, then we have that every asymptotically Euclidean metric is admissible. The horns and cones of [4] that are of the form $g=d r^{2}+r^{2 \alpha} d s_{\partial M_{0}}^{2}$ on $(\omega, r) \in \partial M_{0} \times(0,1)=M_{\infty}$ and with $\alpha \geqslant 1$ are admissible in our sense. To see this, let $z=-\int_{1}^{r} t^{-\alpha} d t$. Then on $(\omega, z) \in \partial M_{0} \times \mathbf{R}^{+}$ $=M_{\infty}$

$$
g=\left\{\begin{array}{l}
{[\exp (-2(\alpha /(\alpha-1)) \ln ((\alpha-1) z+1))]\left(d z^{2}+d s_{\partial M_{0}}^{2}\right), \quad \alpha>1,} \\
e^{-2 z}\left(d z^{2}+d s_{\partial M_{0}}^{2}\right), \quad \alpha=1 .
\end{array}\right.
$$

In both cases $g$ is admissible. If $\rho=-\ln z$ on $\partial M_{0} \times(1, \infty)$ then $g=e^{2 \rho} h$ is admissible and so for instance the cuspidal metrics of [11] are admissible. For more examples see the end of the introduction.

It is for the Laplacians associated with admissible metrics that we prove Fredholm results. Since the manifolds under consideration are noncompact, weighted Sobolev spaces must be used. This is not due to some perversity of the author, but rather out 
of necessity. It just is not the case that

$$
\Delta_{g}: L_{2}^{p}\left(\bigwedge^{q} M, g\right) \rightarrow L^{p}\left(\bigwedge^{q} M, g\right)
$$

is always Fredholm. For instance, if $g$ is asymptotically a product metric on $M_{\infty}$, then (0.2) is Fredholm if and only if $H_{D R}^{q-1}\left(\partial M_{0}\right)=H_{D R}^{q}\left(\partial M_{0}\right)=0$.

In the case of metrics asymptotically translation invariant on $M_{\infty}$, the weights needed are $\exp (\tilde{\delta})$ with $\tilde{\delta} \in C^{\infty}(M ; \mathbf{R})$ and $\tilde{\delta}(\omega, z)=\delta_{l} z$ on the $l$ th end of $M$. What matters about $\tilde{\delta}$ is its values on $M_{\infty}$, and so it may be identified with an element $\delta$ of $\mathbf{R}^{L}$, where $L$ is the number of ends of $M$ and $\tilde{\delta}(\omega, z)=\delta_{l} z$ on the $l$ th end. Using this identification we write our weights as $e^{\delta z}$.

In the case of a general admissible metric, $g=e^{2 \rho} h$, these weights must be modified. It turns out for $g=e^{2 \rho} h$ the appropriate Sobolev spaces are the ones with the norms

$$
\|\sigma\|\left(W_{s, \delta, a}^{p}\left(\wedge^{q} M, g\right)\right)=\left(\sum_{t=0}^{s} \int_{M}\left\|e^{\delta z+(t+a) \rho} D_{(g)}^{t} \sigma\right\|_{g}^{p} d V_{g}\right)^{1 / p}
$$

with $\delta \in \mathbf{R}^{L}, 1<p<\infty, s \in \mathbf{N}$, and $a \in \mathbf{R}$. The associated Sobolev spaces are

$$
W_{s, \delta, a}^{p}\left(\bigwedge^{q} M, g\right)=\left\{\sigma \in L_{s, \mathrm{loc}}^{p}\left(\bigwedge^{q} M\right) \mid\|\sigma\|\left(W_{s, \delta, a}^{p}\left(\bigwedge^{q} M, g\right)\right)<\infty\right\} .
$$

Various properties of the spaces $W_{s, \delta, a}^{p}\left(\wedge^{q} M, g\right)$ are established in $\S \S 3$ and 4 . These include the density of $C_{0}^{\infty}\left(\wedge^{q} M\right)$, a weighted Sobolev embedding theorem, and a compact embedding theorem.

One of our principal Fredholm results is that

$$
\Delta_{g}: W_{s+2, \delta, a}^{p}\left(\bigwedge^{q} M, g\right) \rightarrow W_{s, \delta, a+2}^{p}\left(\wedge^{q} M, g\right)
$$

is continuous for all $\delta$ and Fredholm for almost all $\delta \in \mathbf{R}^{L}$. Since $L^{p}\left(\bigwedge^{q} M, g\right)=$ $W_{0,0,0}^{p}\left(\bigwedge^{q} M, g\right)$ and $W_{0,0,0}^{p}\left(\bigwedge^{q} M, g\right) \subset W_{0, \delta, 0}^{p}\left(\bigwedge^{q} M, g\right)$ for all $\delta \in \mathbf{R}^{L}$ with $\delta_{l} \leqslant 0$, it follows from this Fredholm result and some regularity results for harmonic forms that the space of harmonic forms in $L^{p}\left(\bigwedge^{q} M, g\right)$ is finite dimensional. In fact more generally there is the Liouville theorem that for all $a \in \mathbf{R}$ and $\delta \in \mathbf{R}^{L}$ the space of harmonic forms in $W_{0, \delta, a}^{p}\left(\bigwedge^{q} M, g\right)$ is finite dimensional. This combined with the regularity results for harmonic forms in $\$ 5$ implies that the space of harmonic forms satisfying $\sup _{M}\left\|e^{\delta z} \boldsymbol{\sigma}\right\|_{g}<\infty$ is finite dimensional for each fixed $\delta \in \mathbf{R}^{L}$.

It is still not the case that

$$
\Delta_{g}: W_{2,0,-2}^{p}\left(\bigwedge^{q} M, g\right) \rightarrow W_{0,0,0}^{p}\left(\bigwedge^{q} M, g\right)=L^{p}\left(\bigwedge^{q} M, g\right)
$$

is always Fredholm. However, because the kernel and the kernel of the adjoint of (0.6) are finite dimensional and because (0.5) with $a=-2$ is Fredholm for some $\delta \in \mathbf{R}^{L}$ with $\delta_{l} \leqslant 0$, it is possible to construct a Banach space $\tilde{W}_{2,0,-2}^{p}\left(\wedge^{q} M, g\right)$ such that

$$
\Delta_{g}: \tilde{W}_{2,0,-2}^{p}\left(\wedge^{q} M, g\right) \rightarrow L^{p}\left(\wedge^{q} M, g\right)
$$

is Fredholm. If $(0.6)$ is Fredholm, then $\tilde{W}_{2,0,-2}^{p}\left(\bigwedge^{q} M, g\right)$ turns out to be $W_{2,0,-2}^{p}\left(\wedge^{q} M, g\right)$ with an equivalent norm. In general $W_{2,0,-2}^{p}\left(\bigwedge^{q} M, g\right)$ is dense in $\tilde{W}_{2,0,-2}^{p}\left(\wedge^{q} M, g\right)$ and the latter space is contained in $\bigcap_{\eta<0} W_{2, \eta,-2}^{p}\left(\wedge^{q} M, g\right)$. This along with the proof of the other Fredholm result is done in $\S 5$. The analogous results for $d+d_{g}^{*}$ are also proved there. 
Because of these Fredholm results

$$
L^{p}\left(\bigwedge^{q} M, g\right)=\Delta_{g}\left(\tilde{W}_{2,0,-2}^{p}\left(\bigwedge^{q} M, g\right)\right) \oplus F
$$

with $\operatorname{dim} F=\operatorname{dimension}$ of the space of harmonic forms in $L^{p^{\prime}}\left(\bigwedge^{q} M, g\right)\left(1 / p^{\prime}+\right.$ $1 / p=1)$. In $\S 6$ by using the weighted Sobolev imbedding theorem we provide sufficient conditions for $F$ to consist of harmonic forms. In fact this is done for the general space $W_{s, \delta, a}^{p}\left(\wedge^{q} M, g\right)$. A consequence of these results is that on an orientable manifold if $g$ is admissible and $\phi$ is a function such that $e^{\delta z} \phi \in L^{p}(M, g)$ for $\delta_{l}$ sufficiently negative for each $l$, then $\phi=d_{g}^{*} \sigma$ for some 1 -form

$$
\sigma \in \bigcap_{\tau<\delta} W_{0, \tau,-1}^{p}\left(\wedge^{1} M, g\right)
$$

In fact this is true even if $g$ is only equivalent to an admissible metric; i.e., there is an admissible metric $g_{1}$ and $c \in \mathbf{R}^{+}$such that $g / c \leqslant g_{1} \leqslant c g$.

In $\$ 7$ we turn our attention to the properties of the space of closed and coclosed forms in $L^{2}\left(\bigwedge^{q} M, g\right)$ for metrics equivalent to admissible metrics. This space is denoted $\tilde{\mathscr{S}}^{2}\left(\bigwedge^{q} M, g\right)$. Note that in the case of complete metrics, $\tilde{\mathfrak{E}}^{2}\left(\bigwedge^{q} M, g\right)$ is the same as the space of $L^{2}$-harmonic forms.

Qne of the chief results of the section is that if a metric (not necessarily equivalent to an admissible one) is equivalent to one that is $q$-bounded above (see 7.3), then $\tilde{\mathfrak{S}}^{2}\left(\wedge^{q} M, g\right)$ contains no exact elements. Thus $\operatorname{dim} \tilde{\mathscr{S}}^{2}\left(\wedge^{q} M, g\right) \leqslant \operatorname{dim} H_{D R}^{q}(M)$ and each element of $\tilde{\mathfrak{E}}^{2}\left(\wedge^{q} M, g\right)$ is a unique closed and coclosed representative of a class in $H_{D R}^{q}(M)$.

Here and throughout the paper

$$
H_{D R}^{q}(M)=\left[\operatorname{Ker} d: C^{\infty}\left(\wedge^{q} M\right) \rightarrow C^{\infty}\left(\wedge^{q+1} M\right)\right] / d C^{\infty}\left(\wedge^{q-1} M\right) .
$$

There are many examples of metrics that are $q$-bounded above. For instance, if $h$ is asymptotically translation invariant, then it is $q$-bounded above for all $q$. If $g=e^{2 \phi} h$ is conformal to $h$ ( $g$ not necessarily admissible) and $\phi(\omega, z)$ is increasing on $M_{\infty}$ for each $\omega \in \partial M_{0}$, then $g$ is $q$-bounded above for $q \geqslant n / 2$; if $\phi$ is decreasing, then $g$ is $q$-bounded above for $q \leqslant n / 2$.

When $g$ is equivalent to an admissible metric, the Fredholm results for $d+d_{g}^{*}$ mentioned above imply that every class of $H_{D R}^{q}(M)$ with a representative in $L^{2}\left(\bigwedge^{q} M, g\right)$ has a representative in $\tilde{\mathfrak{S}}^{2}\left(\bigwedge^{q} M, g\right)$. Thus the question arises as to which cohomology classes have representatives in $L^{2}\left(\wedge^{q} M, g\right)$. This is determined in (7.9).

For $g$ a metric and $\Omega_{1}, \ldots, \Omega_{L}$ the components of $\partial M_{0}$ let

$$
\text { (0.10) } \mathscr{A}=\mathscr{A}(g ; q)=\left\{l \mid \int_{\Omega_{l} \times \mathbf{R}^{+}}\|\phi\|_{g}^{2} d V_{g}=\infty \text { for some } \phi \in C^{\infty}\left(\wedge^{q} M\right)_{\mathbf{R}^{+}}\right\}
$$

where $C^{\infty}\left(\wedge^{q} M\right)_{\mathbf{R}^{+}}$is the space of $C^{\infty} q$-forms that are $\mathbf{R}^{+}$invariant on $M_{\infty}$. Also let $M_{\infty}^{\infty}(g ; q)=\bigcup_{l \in \mathscr{A}} \Omega_{l} \times \mathbf{R}^{+}$. It is shown in (7.9) that if $g$ is equivalent to an admissible metric, then the classes in $H_{D R}^{q}(M)$ with a representative in $\tilde{\mathfrak{S}}^{2}\left(\bigwedge^{q} M, g\right)$ are exactly those with compact support on $M_{\infty}^{\infty}(g ; q)$; i.e. have a representative that is 0 on $\bigcup_{l \in \mathscr{A}} \Omega_{l} \times[R, \infty)$ for some $R>0$. If in addition $g$ is $q$-bounded above, then the representation is unique. 
In the last section we look at some properties of the $L^{2}$-cohomology (see Definition (8.2)) of the Riemannian manifolds which are considered in this paper. In particular, if $M$ is a manifold with finitely many ends, equipped with a metric $g$ that is equivalent to an admissible one, and $H_{2}^{q}(M)$ is the $L^{2}$-cohomology of $M$, then we present some results concerning when the natural map

$$
i: \tilde{\mathfrak{S}}^{2}\left(\bigwedge^{q} M, g\right) \rightarrow H_{2}^{q}(M)
$$

is $1-1$, is onto, is an isomorphism. When it is an isomorphism we say that the Strong Hodge Theorem holds (see [4]). When (0.11) is not onto then we have the interesting fact that $H_{2}^{q}(M)$ is infinite dimensional.

For example, an easy result (Proposition (8.5)) is that if $g$ is equivalent to a metric that is $q$-bounded above, then (0.11) is 1-1. A more difficult result (Theorem (8.7)) is that if $g$ is equivalent to an admissible metric $g_{1}=e^{2 \rho} h$ and $\rho$ is bounded below, then $(0.11)$ is 1-1. Moreover, if $\lim _{\sup _{z \rightarrow \infty}} \rho(\omega, z)=\infty$ on at least one end of $M$, then $(0.11)$ is not onto for $q>0$.

In Theorem (8.9) we treat the case in which $g$ is equivalent to an admissible metric $g_{1}=e^{2 \rho} h$ and $\rho$ is bounded above. One of the results of that theorem is that if $\rho \geqslant(\varepsilon-1) \ln z$ on $\partial M_{0} \times[1, \infty)$ for some $0<\varepsilon<1$ and $\rho$ is slowly oscillating in the sense of (8.8) then (0.11) is onto if and only if $H_{D R}^{q-1}\left(\partial M_{0}\right)=0$. For example, if $\rho$ is bounded, then either the Strong Hodge Theorem holds or, when $H_{D R}^{q-1}\left(\partial M_{0}\right) \neq 0$, $H_{2}^{q}(M)$ is infinite dimensional (also see [13] for this result for bounded $\rho$ ).

The condition that $\rho \geqslant(\varepsilon-1) \ln z$ for some $0<\varepsilon<1$ is in some sense sharp for the necessity of $H_{D R}^{q-1}\left(\partial M_{0}\right)$ being 0 for $(0.11)$ to be onto. If $g$ is $q$-bounded above and $z e^{\rho}$ is bounded on $\partial M_{0} \times \mathbf{R}^{+}$, then the Strong Hodge Theorem holds regardless of what $H_{D R}^{q}\left(\partial M_{0}\right)$ is (see (8.9)). Thus, for instance, this is true of the cuspidal metrics of [11].

Finally, if $\rho$ is $O(z)$ but not $o(z)$ as $z \rightarrow \infty$ on $\partial M_{0} \times \mathbf{R}^{+}$, then $(0.11)$ is onto. This is the case, for example, for the metric cones in [4].

To illustrate the scope of these results, four examples will now be given. In all the examples there is the orthogonal decomposition

$$
L^{2}\left(\bigwedge^{q} M, g\right)=d \tilde{\tilde{W}}_{1,0,-1}^{2}\left(\bigwedge^{q-1} M, g\right) \oplus d_{g}^{*} \tilde{W}_{1,0,-1}^{2}\left(\bigwedge^{q+1} M, g\right) \oplus \tilde{\mathfrak{S}}^{2}\left(\bigwedge^{q} M, g\right)
$$

In the cases in which $g$ is complete, $\tilde{\mathfrak{S}}^{2}\left(\bigwedge^{q} M, g\right)$ contains all harmonic, $L^{2}$ forms and so

$$
\Delta_{g}\left(\tilde{W}_{2,0,-2}^{2}\left(\bigwedge^{q} M, g\right)\right)=d \tilde{\tilde{W}}_{1,0,-1}^{2}\left(\bigwedge^{q-1} M, g\right) \oplus d_{g}^{*} W_{1,0,-1}^{2}\left(\bigwedge^{q+1} M, g\right)
$$

EXAMPLE (0.14). $g$ is asymptotically translation invariant.

In this case $g$ is $q$-bounded above for all $q$ and admissible. Furthermore $M_{\infty}^{\infty}(g ; q)=M_{\infty}$ for all $q$. Thus

$$
\tilde{\mathscr{S}}^{2}\left(\bigwedge^{q} M, g\right) \approx \text { image of } H_{\text {comp }}^{q}(M) \text { in } H_{D R}^{q}(M) \text { for all } q
$$

and provides unique representatives for that image. Also $H_{2}^{q}(M)$ is infinite dimensional if $H_{D R}^{q-1}\left(\partial M_{0}\right) \neq 0$. Otherwise, $H_{2}^{q}(M) \approx \tilde{\mathfrak{S}}^{2}\left(\bigwedge^{q} M, g\right)$. 
EXAMPLE (0.15). $h$ is asymptotically translation invariant, $g=e^{2 \rho} h$ is admissible, $\rho(\omega, z)$ is increasing on $M_{\infty}$ for each $\omega \in \partial M_{0}$, and $\rho(\omega, z)>[(1+\varepsilon) / 2] \ln z$ on $\partial M_{0} \times[1, \infty)$ for some $\varepsilon>0$.

In this case $g$ is $q$-bounded above for $q \geqslant n / 2$ and admissible. Furthermore $M_{\infty}^{\infty}(g, q)=M_{\infty}$ if $q \leqslant n / 2$. However, if $q>n / 2$ then $M(g, q)$ is empty. Thus

$$
\tilde{\mathfrak{S}}^{2}\left(\wedge^{q} M, g\right) \approx\left\{\begin{array}{l}
H_{D R}^{q}(M) \text { for } q>n / 2, \\
\text { image of } H_{\text {comp }}^{q}(M) \text { in } H_{D R}^{q}(M) \text { if } q=n / 2
\end{array}\right.
$$

and provides unique representatives for these spaces. For $q<n / 2$ only the image of $H_{\text {comp }}^{q}(M)$ in $H_{D R}^{q}(M)$ has representatives in $\tilde{\mathscr{S}}^{2}\left(\bigwedge^{q} M, g\right)$. Moreover the representatives need not be unique. For example, if $M$ is orientable, then obviously $\tilde{\mathfrak{S}}^{2}\left(\wedge^{q} M, g\right)={ }_{g} \tilde{\mathfrak{S}}^{2}\left(\bigwedge^{n-q} M, g\right)$ and so $\operatorname{dim} \tilde{\mathfrak{S}}^{2}\left(\wedge^{q} M, g\right)=\operatorname{dim} H_{\text {comp }}^{q}(M)$ for $q<$ $n / 2$. Also $\operatorname{dim} H_{2}^{q}(M)=\infty$ if $q>0$.

EXAMPLE (0.16). $h$ is asymptotically translation invariant, $g=e^{2 \rho} h$ is admissible, $\rho(\omega, z)$ decreasing, and $\rho(\omega, z)<-[(1+\varepsilon) / 2] \ln z$ on $\partial M_{0} \times[1, \infty)$ for some $\varepsilon>0$.

This case is exactly the same as the previous one except that all the inequalities for $q$ are reversed. Thus

$$
\tilde{\mathfrak{S}}^{2}\left(\wedge^{q} M, g\right) \approx\left\{\begin{array}{l}
H_{D R}^{q}(M) \text { for } q<n / 2, \\
\text { image of } H_{\text {comp }}^{q}(M) \text { in } H_{D R}^{q}(M) \text { if } q=n / 2
\end{array}\right.
$$

and provides unique representatives for these spaces. For $q>n / 2$ only the image of $H_{\text {comp }}^{q}(M)$ in $H_{D R}^{q}(M)$ has representatives in $\tilde{\mathscr{S}}^{2}\left(\wedge^{q} M, g\right)$. Moreover the representative need not be unique. If $M$ is orientable, then $\operatorname{dim} \tilde{\mathfrak{S}}^{2}\left(\wedge^{q} M, g\right)=\operatorname{dim} H_{\text {comp }}^{q}(M)$ for $q>n / 2$. Also if $\varepsilon>1$, then $H_{2}^{q}(M) \simeq \tilde{\mathfrak{F}}^{2}\left(\bigwedge^{q} M, g\right)$ for $q \leqslant n / 2$. If moreover $\rho$ is $O(z)$ but not $o(z)$ as $z \rightarrow \infty$ on $\partial M_{0} \times \mathbf{R}^{+}$, then $\operatorname{dim} H_{2}^{q}(M) \leqslant \operatorname{dim} \tilde{\mathfrak{F}}^{2}\left(\bigwedge^{q} M, g\right)$ for $q>n / 2$.

EXAMPLE (0.17). $h$ is asymptotically translation invariant, $g=e^{2 \rho} h$ and $\rho(\omega, z)$ $=(\sin (\ln z)) \ln z$ on $\partial M_{0} \times[1, \infty)$.

Here $g$ is admissible; however, it is $q$-bounded above only for $q=n / 2$ (if $n$ is even). Moreover $M_{\infty}^{\infty}(g ; q)=M_{\infty}$ for all $q$. Thus only the image of $H_{\text {comp }}^{q}(M)$ in $H_{D R}^{q}(M)$ has representatives in $\tilde{\mathscr{S}}^{2}\left(\wedge^{q} M, g\right)$. If $q=n / 2$ these representatives are unique. For $q \neq n / 2$ our techniques do not allow us to say anything more; except, of course, if $q=0$ or $n$, which are trivial cases.

As we noted before, Example (0.15) contains all asymptotically Euclidean metrics on manifolds that outside $M_{0}$ are diffeomorphic to a finite number of copies of $\mathbf{R}^{n}$ minus a ball. Similarly Example (0.17) contains the metric cones and horns in [4] and cusps in [11]. Also note that if $g$ is equivalent to any of the metrics in these examples, then everything said about the space of closed and coclosed $L^{2}$ forms for the metric that $g$ is equivalent to is also true for $\tilde{\mathfrak{S}}^{2}\left(\bigwedge^{q} M, g\right)$. In fact one can even get a version of the decomposition (0.12) when $M$ is oriented. More specifically, if $g$ is equivalent to an admissible metric $g_{1}$, then

(0.18) $\quad L^{2}\left(\wedge^{q} M, g\right)=d \tilde{\tilde{W}}_{1,0,-1}^{2}\left(\wedge^{q-1} M, g_{1}\right) \oplus d_{g}^{*} B \oplus \tilde{\mathscr{S}}^{2}\left(\bigwedge^{q} M, g\right)$

where $B=* \tilde{\tilde{W}}_{1,0,-1}^{2}\left(\bigwedge^{n-q-1} M, g_{1}\right)$ and the norm on $B$ is

$$
\|\sigma\|_{B}=\left\|*_{g} \sigma\right\|\left(W_{1,0,-1}^{2}\left(\wedge^{n-q-1} M, g_{1}\right)\right) \text {. }
$$


This follows easily from (0.12) and Kodaira's theorem.

Finally there is the question as to whether one needs to use weighted Sobolev spaces in order to get Fredholm results for $\Delta_{g}$ and $d_{g}+d_{g}^{*}$ for admissible $g$. The answer is yes. For instance, suppose $B$ is a Banach space of $q$-forms with the properties that $C_{0}^{\infty}\left(\wedge^{q} M\right)$ is dense in $B$ and $\Delta_{g}: B \rightarrow L^{p}\left(\wedge^{q} M, g\right)$ is Fredholm. Since $\Delta_{g}: B \rightarrow L^{p}\left(\bigwedge^{q} M, g\right)$ is Fredholm, the norm on $B$ is equivalent to $\left\|\Delta_{g} \sigma\right\|_{p}+$ $\left\|\pi_{B} \sigma\right\|$ where $\pi_{B}$ is a projection of $B$ onto $\operatorname{Ker} \Delta_{g} \cap B=K_{1}$. Similarly the norm on $\tilde{W}_{2,0,-2}^{p}\left(\bigwedge^{q} M, g\right)$ is equivalent to $\left\|\Delta_{g} \sigma\right\|_{p}+\|\tilde{\pi} \sigma\|$ with $\tilde{\pi}$ a projection of $\tilde{W}_{2,0,-2}^{p}\left(\bigwedge^{q} M, g\right)$ onto

$$
\operatorname{Ker} \Delta_{g} \cap \tilde{W}_{2,0,-2}^{p}\left(\wedge^{q} M, g\right)=K_{2}
$$

Thus if $F=B \cap \tilde{W}_{2,0,-2}^{p}\left(\bigwedge^{q} M, g\right)$ has norm $\|\sigma\|_{F}=\max \left(\|\sigma\|_{B},\|\sigma\|\left(\tilde{W}_{2,0,-2}^{p}\left(\bigwedge^{q} M, g\right)\right)\right.$, then \|\|$_{F}$ is equivalent to $\left\|\Delta_{g} \sigma\right\|_{p}+\|\pi \sigma\|$ where $\pi=\left(\pi_{B}, \pi\right): F \rightarrow K_{1} \oplus K_{2}$. It follows from this and the fact that $C_{0}^{\infty}\left(\wedge^{q} M\right) \subset F$ that $\Delta_{g}: F \rightarrow L^{p}\left(\wedge^{q} M, g\right)$ is Fredholm, which in turn means that as sets $B=\tilde{W}_{2,0,-2}^{p}\left(\bigwedge^{q} M, g\right)$ and that the norms are equivalent.

1. Notation. In this section we list some of the notation used that is not defined in the text. First of all $\mathbf{R}$ is the real numbers, $\mathbf{R}^{+}=\{z \in \mathbf{R} \mid z>0\}$, and $\mathbf{N}$ is the nonnegative integers.

As usual $T_{r}^{q}(M)$ is the bundle of $(r, q)$ tensors over $M, \wedge^{q} M$ is the bundle of exterior $q$-forms (we often think of $\wedge^{q} M$ as being in $T_{0}^{q} M$ ), and $\wedge^{*} M=\oplus_{q=0}^{n} \wedge^{q} M$. If $E$ is any of these bundles, then $\Gamma(E)$ is the space of all sections of $E$ and $C^{\infty}(E)$ is the space of $C^{\infty}$ sections; $C^{\infty}(M)$ is the $C^{\infty}$ functions on $M$.

By a metric we always mean a $C^{\infty}$, Riemannian metric, though many of the results hold for metrics with much less regularity. For a metric, $g$, we let $D_{(g)}$ be the covariant derivative associated with $g$ by the Levi-Civita connection. If $t \in \mathbf{N}$, then $D_{(g)}^{t}=D_{(g)} \cdots D_{(g)} t$ times. On the other hand $\nabla_{i}$ is, as usual, the covariant derivative in the $i$ th coordinate direction. In this regard $\nabla^{i}=g^{i j} \nabla_{j}$. Also, we take \|\|$_{g(x)}$ to be the norm on $\left.T_{r}^{q} M\right|_{x}$, or $\left.\wedge^{q} M\right|_{x}$, or $\left.\wedge^{*} M\right|_{x}$ induced by $g(x)$.

For any metric, $g$, on $M, s \in \mathbf{N}$, and $1 \leqslant p<\infty$ and for $E=T_{r}^{q} M, \wedge^{q} M$, or $\wedge^{*} M$, we let

$$
L_{s}^{p}(E, g)=\left\{\sigma \in \Gamma(E) \mid\left(\sum_{t=0}^{s} \int_{M}\left\|D_{(g)}^{t} \sigma\right\|_{g}^{p} d V_{g}\right)^{1 / p}<\infty\right\}
$$

where $d V_{g}$ is the volume measure arising from $g$. Also we take

$$
L_{s, \mathrm{loc}}^{p}(E)=\left\{\sigma \in \Gamma(E) \mid \phi \sigma \in L_{s}^{p}(E) \text { for all } \phi \in C_{0}^{\infty}(M)\right\} .
$$

Note that $L_{s, \text { loc }}^{p}(E)$ is independent of the metric.

Lastly, if $g$ is a metric, then $d_{g}^{*}$ is the operator from $C^{\infty}\left(\bigwedge^{q} M\right)$ to $C^{\infty}\left(\wedge^{q-1} M\right)$ defined locally by

$$
\left(d_{g}^{*} \alpha\right)_{i, \ldots, i_{q-1}}=-\nabla^{j} \alpha_{j i_{1} \cdots i_{q-1}} .
$$

Thus it is the formal adjoint of $d$. 
2. Admissible metrics on manifolds with finitely many ends. Let $M$ be a manifold with finitely many ends. Recall this means that $M$ contains a compact manifold, $M_{0}$, with boundary; that $M-M_{0}=\partial M_{0} \times(0, \infty)$, and that $\partial M_{0}$ has only finitely many components. There is an obvious $\mathbf{R}^{+}$-action on $M_{\infty} \equiv \partial M_{0} \times \mathbf{R}^{+}$; namely $(\omega, z) \mapsto\left(\omega, z+z_{0}\right)$ for $z_{0} \in \mathbf{R}^{+}$. We say that a metric on $M$ is translation invariant, if on $M_{\infty}$ it is invariant under this $\mathbf{R}^{+}$-action.

Definition (2.1). Suppose $h_{\infty}$ is a translation invariant metric, with covariant derivative $D_{\infty}$. A metric, $h$, is asymptotic to $h_{\infty}$ if for each $t \in \mathbf{N}$

$$
\lim _{z \rightarrow \infty} \sup _{\omega \in \partial M_{0}}\left\|D_{\infty}^{t} h(\omega, z)-D_{\infty}^{t} h_{\infty}(\omega)\right\|_{h_{\infty}}=0 .
$$

If $h$ is asymptotic to a translation invariant metric, then $h$ is said to be asymptotically translation invariant.

CONVENTION (2.2). Henceforth $h$ will always denote an asymptotically translation invariant metric.

Definition (2.3). Let $g=e^{2 \rho} h$ with $\rho \in C^{\infty}(M)$. The metric $g$ is admissible if there is a $C^{\infty}, \mathbf{R}^{+}$-invariant 1 -form, $\theta$, on $M_{\infty}$ with the property

$$
\lim _{z \rightarrow \infty} \sup _{\omega \in \partial M_{0}}\left\|D_{(h)}^{t+1} \rho-D_{(h)}^{t} \theta\right\|_{h}=0 .
$$

A few remarks about these definitions are in order. First of all note that all geometric quantities associated with a metric, $h$, that is asymptotic to a translation invariant metric, $h_{\infty}$, asymptotically become those of $h_{\infty}$. In fact one has the following

Proposition (2.4). Let $h_{\infty}$ be translation invariant and $h$ asymptotic to $h_{\infty}$. Let $A$ be the tensor field $\Gamma_{(h) j k}^{i}-\Gamma_{\left(h_{\infty}\right) j k}^{i}$. Then

$$
\lim _{z \rightarrow \infty} \sup _{\omega \in \partial M_{0}}\left\|D_{\infty}^{t} A\right\|_{h_{\infty}}=0
$$

for all $t \in \mathbf{N}$. Furthermore if $X \in C^{\infty}\left(T_{r}^{q} M\right)$ satisfies

$$
\sup _{M}\left\|D_{\infty}^{t} X\right\|_{h_{\infty}}<\infty
$$

for all $t$, then

$$
\lim _{z \rightarrow \infty} \sup _{\partial M_{0}}\left\|D_{(h)}^{t} X-D_{\infty}^{t} X\right\|_{h_{\infty}}=0
$$

for all $t$.

Proof. Since

$$
A_{j k}^{i}=\frac{1}{2} h^{i l}\left[\left(D_{\infty} h\right)_{k i l}+\left(D_{\infty} h\right)_{j l k}-\left(D_{\infty} h\right)_{l j k}\right],
$$

(2.4.1) follows from (2.1.1). As for (2.4.3) we have for $l \geqslant 0$ that

$$
\begin{aligned}
\left\|D_{(h)}^{l+1} X-D_{\infty}^{l+1} X\right\|_{h_{\infty}} \leqslant & \left\|D_{(h)}^{l}\left(D_{(h)} X-D_{\infty} X\right)-D_{\infty}^{l}\left(D_{(h)} X-D_{\infty} X\right)\right\|_{h_{\infty}} \\
& +\left\|D_{\infty}^{l}\left(D_{(h)} X-D_{\infty} X\right)\right\|_{h_{\infty}}+\left\|D_{(h)}^{l} D_{\infty} X-D_{\infty}^{l} D_{\infty} X\right\|_{h_{\infty}} .
\end{aligned}
$$


Clearly if $X$ satisfies (2.4.2), then so does $D_{\infty} X$. Furthermore because of (2.4.1) and the fact that $D_{(h)} X-D_{\infty} X$ is a sum of contractions of $\pm A \otimes X$, we have that $D_{(h)} X-D_{\infty} X$ also satisfies (2.4.2) when $X$ does. Thus (2.4.3) holds for $t=l+1$ if it holds for $t=l$. Since (2.4.3) is obviously true for $l=0$, we are done.

Another thing to note is that the norm and covariant derivative in (2.1.1) do not have to be those of $h_{\infty}$.

Proposition (2.5). Let $h$, $j$, and $k$ be asymptotically translation invariant metrics. If $X \in C^{\infty}\left(T_{r}^{q} M\right)$ satisfies

$$
\lim _{z \rightarrow \infty} \sup _{\omega \in \partial M_{0}}\left\|D_{(h)}^{t} X\right\|_{h}=0
$$

for all $t$, then

$$
\lim _{z \rightarrow \infty} \sup _{\omega \in \partial M_{0}}\left\|D_{(j)}^{t} X\right\|_{k}=0
$$

for all $t$.

Proof. Because $h$ and $k$ are asymptotically translation invariant, there is a constant, $c$, such that $c^{-1}\|\|_{k} \leqslant\|\|_{h} \leqslant c\|\|_{k}$. Thus (2.5.1) implies (2.5.2) for $t=0$.

If $l \geqslant 0$, then

$$
\left\|D_{(j)}^{l+1} X\right\|_{k} \leqslant\left\|D_{(j)}^{l} D_{(h)} X\right\|_{k}+\sum_{i=1}^{3}\left\|D_{(j)}^{l}\left(D_{(i)}-D_{(i+1)}\right) X\right\|_{k}
$$

with $D_{(1)}=D_{(j)}, D_{(2)}=D_{\left(j_{\infty}\right)}, D_{(3)}=D_{\left(h_{\infty}\right)}$, and $D_{(4)}=D_{(h)}$.

Let $B_{(i)}=D_{(i)}-D_{(i+1)}$. Then $D_{(i)} X-D_{(i+1)} X$ is a sum of contractions of $\pm B_{(i)} \otimes X$, and so by Leibnitz's rule

$$
\begin{aligned}
\left\|D_{(j)}^{l+1} X\right\|_{k} \leqslant & \left\|D_{(j)}^{l} D_{(h)} X\right\|_{k}+c \sum_{s=0}^{l}\left\|D_{(j)}^{s} B_{1}\right\|_{k}\left\|D_{(j)}^{l-s} X\right\|_{k} \\
& +c \sum_{s=0}^{l}\left\|D_{(j)}^{s} B_{2}\right\|_{k}\left\|D_{(j)}^{l-s} X\right\|_{k}+\left\|D_{(j)}^{l}\left(D_{\left(h_{\infty}\right)} X-D_{(h)} X\right)\right\|_{k}
\end{aligned}
$$

It follows from the previous proposition that $D_{\left(h_{\infty}\right)} X-D_{(h)} X$ satisfies (2.5.1) for all $t$ and that $\sup _{M}\left\|D_{(j)}^{s} B_{1}\right\|_{j}<\infty$ for all $s$. Since $B_{(2)}$ is independent of $z$ on $\partial M_{0} \times \mathbf{R}^{+}$it also follows from (2.4) that $\sup _{M}\left\|D_{(j)}^{s} B_{2}\right\|_{j}<\infty$ for all $s$. Because $D_{(h)} X$ obviously satisfies (2.5.1) when $X$ does and the $j$ and $k$ norms are equivalent, we have that (2.5.2) holds for $t=l+1$ if it holds for $t=l$. Since it holds for $t=0$ we are done.

It is also useful to note that if $g=e^{2 \rho} h$ is admissible, then $\rho$ satisfies (2.3.1) for some $\theta$.

Proposition (2.6). Suppose $g=e^{2 \rho} h$ is admissible. Then $\rho$ satisfies (2.3.1) for some $\theta$.

Proof. By assumption there exists a metric, $\bar{h}$, asymptotic to a translation invariant metric, $\bar{h}_{\infty}$; a function, $\bar{\rho}$; and a translation invariant 1 -form, $\bar{\theta}$, such that $g=e^{2(\bar{\rho})} \bar{h}$ and (2.3.1) holds for $\bar{\rho}$ and $\bar{\theta}$. Hence $h=e^{2(\bar{\rho}-\rho)} \bar{h}$. By (2.1.1) and the 
previous proposition this means that

$$
\lim _{z \rightarrow \infty} \sup _{\omega \in \partial M_{0}}\left\|D_{(j)}^{t}\left(e^{2(\bar{\rho}-\rho)} \bar{h}-h_{\infty}\right)\right\|_{j}=0
$$

for all $t$ and all asymptotically translation invariant metrics $j$. It follows that $\lim _{z \rightarrow \infty}(\bar{\rho}(\omega, z)-\rho(\omega, z))$ exists for each $\omega \in \partial M_{0}$, call it $\phi(\omega)$, and that $h_{\infty}=$ $e^{2 \phi} \bar{h}_{\infty}$. Thus $\phi$ is $C^{\infty}$. Picking $j=\bar{h}_{\infty}$ in (2.6.1) we get

$$
\lim _{z \rightarrow \infty} \sup _{\omega \in \partial M_{0}}\left\|D_{\left(h_{\infty}\right)}^{t}\left(e^{2(\bar{\rho}-\rho)} \bar{h}-e^{2 \phi} \bar{h}_{\infty}\right)\right\|_{\bar{h}_{\infty}}=0 .
$$

It is easy to see from this that $\rho$ satisfies (2.3.1) with $\boldsymbol{\theta}=\overline{\boldsymbol{\theta}}-d \phi$.

Several examples of admissible metrics were given in the introduction. In these examples the exponent of the conformal factor, $\rho$, is a function of only $z$ on $M_{\infty}$ and is $O(z)$ as $z \rightarrow \infty$ on each component of $M_{\infty}$. We now show that every admissible metric is of this form.

Lemma (2.7). Suppose $\rho$ and $\theta$ are as in (2.3). If we express $\theta$ as $\theta=\psi(\omega)+f(\omega) d z$ with $\psi \in C^{\infty}\left(\wedge^{1} \partial M_{0}\right)$, then $f$ is locally constant on $\partial M_{0}$.

Proof. Let $\rho=f(\omega) z+\sigma(\omega, z)$ on $M_{\infty}$. Since

$$
d \rho=\left(f(\omega)+\partial_{z} \sigma(\omega, z)\right) d z+z \tilde{d} f+\tilde{d} \sigma,
$$

where $\tilde{d}$ is the exterior derivative on $\partial M_{0}$, we have by (2.3.1) that

$$
\lim _{z \rightarrow \infty} \sup _{\omega \in \partial M_{0}}\left|\partial_{z} \sigma(\omega, z)\right|=0 \text {. }
$$

Let $\gamma:[0,1] \rightarrow \partial M_{0}$ be a path from $\omega_{0}$ to $\omega_{1}$. By Stokes's theorem and (2.7.2) we get

$$
\left|f\left(\omega_{1}\right)-f\left(\omega_{0}\right)\right|=\lim _{z \rightarrow \infty}\left|\int_{\gamma} \tilde{d} \partial_{z} \rho\right|
$$

Since $h$ is asymptotically translation invariant, (2.7.3) combined with the CauchySchwarz inequality gives

$$
\left|f\left(\omega_{1}\right)-f\left(\omega_{0}\right)\right| \leqslant c \lim _{z \rightarrow \infty} \sup _{\omega \in \partial M_{0}}\left\|\tilde{d} \partial_{z} \rho(\omega, z)\right\|_{h} .
$$

However this last term is no greater than $\lim _{z \rightarrow \infty} \sup _{\omega \in \partial M_{0}}\left\|\partial_{z} d \rho(\omega, z)\right\|_{h}$ and by (2.3.1) this is 0 .

Lemma (2.8). Let $\theta=\psi(\omega)+f(\omega) d z$, as above. Then $\psi$ is an exact 1-form on $\partial M_{0}$.

Proof. As in the previous lemma, let $\rho=f(\omega) z+\sigma(\omega, z)$ on $M_{\infty}$. From that lemma we have that $d \rho=f(\omega) d z+d \sigma(\omega, z)$. Hence by condition (2.3.1)

$$
\lim _{z \rightarrow \infty} \sup _{\omega \in \partial M_{0}}\|d \sigma-\psi\|_{h}=0 \text {. }
$$

If $\gamma:[0,1] \rightarrow \partial M_{0}$ is any smooth loop, then

$$
\left|\int_{\gamma} \psi\right|=\left|\int_{\gamma} \psi-d \sigma(\omega, z)\right| \leqslant c \sup _{\partial M_{0}}\|\psi(\omega)-d \sigma(\omega, z)\|_{h} .
$$

Since this is true for every $z$ and (2.8.1) is true, $\int_{\gamma} \psi=0$. 
THeOREM (2.9). Suppose $g=e^{2 \rho} h$ is admissible. There is a $C^{\infty}$ function $\bar{\rho}: M \rightarrow \mathbf{R}$ and an asymptotically translation invariant metric $\bar{h}$ such that

$$
\bar{\rho} \text { is a function of only } z \text { on } M_{\infty} \text {; }
$$

if $\Omega_{1}, \ldots, \Omega_{L}$ are the components of $\partial M_{0}$, then there are constants $c_{1}, \ldots, c_{L}$ such that $\lim _{z \rightarrow \infty} \partial_{z} \bar{\rho}(z)=c_{l}$ uniformly on $\Omega_{\text {; }}$

and

$$
\lim _{z \rightarrow \infty} \partial_{z}^{t} \bar{\rho}(z)=0 \text { uniformly on } \partial M_{0} \text { for all } t>1
$$

Proof. For each $l=1, \ldots, L$ let $\omega_{l}$ be a fixed point in $\Omega_{l}$. We take $\bar{\rho}$ to be any $C^{\infty}$ function on $M$ that equals $\rho\left(\omega_{l}, z\right)$ on $\Omega_{l} \times \mathbf{R}^{+}$and take $\bar{h}$ to be $e^{2(\rho-\bar{\rho})} h$.

If, as in (2.7) and (2.8), $\rho$ is expressed on $M_{\infty}$ as $\rho=f(\omega) z+\sigma(\omega, z)$, where $\theta=\psi(\omega)+f(\omega) d z$, then on $\Omega_{l} \times \mathbf{R}^{+} \bar{\rho}=f\left(\omega_{l}\right) z+\sigma\left(\omega_{l}, z\right)$. That $\bar{\rho}$ satisfies (2.9.2) and (2.9.3) follows from condition (2.3.1).

To show that $\bar{h}$ is asymptotically translation invariant, first note that by Lemma (2.8) there is a function $k(\omega)$ such that $\psi=d k(\omega)$. Combining this with the fact that $f$ is locally constant, we have that if $\gamma:[0,1] \rightarrow \Omega_{t}$ is a path from $\omega_{l}$ to $\omega$ then

$$
\rho(\omega, z)-\rho\left(\omega_{l}, z\right)=\int_{\gamma} d k(\omega)+\int_{\gamma}[d \sigma(\omega, z)-d k(\omega)] .
$$

This means that

$$
\lim _{z \rightarrow \infty}\left|\rho(\omega, z)-\bar{\rho}(z)-\left(k(\omega)-k\left(\omega_{l}\right)\right)\right| \leqslant c \lim _{z \rightarrow \infty} \sup _{\omega \in \partial M_{0}}\|d \sigma-d k\|_{h} .
$$

From condition (2.3.1) we have that for each $t \geqslant 0$

$$
\lim _{z \rightarrow \infty} \sup _{\omega \in \partial M_{0}}\left\|D_{(h)}^{t+1} \sigma-D_{(h)}^{t} d k\right\|_{h}=0 .
$$

Thus the right-hand side of (2.9.6) is 0 and so

$$
\lim _{z \rightarrow \infty} \sup _{\omega \in \partial M_{0}}\left\|\rho(\omega, z)-\bar{\rho}(z)-\left(k(\omega)-k\left(\omega_{l}\right)\right)\right\|_{h}=0 .
$$

Combining this with (2.9.7) and the fact that $h$ is asympotic to a translation invariant $h_{\infty}$, we get that $\bar{h}$ is asympotic to $e^{2(k(\omega)-j(\omega))} h_{\infty}$ where $j(\omega)=k\left(\omega_{l}\right)$ on $\Omega_{l}$.

That $\bar{\rho}$ satisfies condition (2.3.1) for some $\bar{\theta}$ follows from Proposition 2.6. ( $\bar{\theta}$ in fact is $f(\omega) d z$.)

We conclude this section with a lemma which will be useful in the next two sections.

Lemma (2.10). Let $g$ be an admissible metric. If $T \in C^{\infty}\left(T_{r}^{q} M\right)$, then in terms of a coordinate system of the form $\left(\Omega_{\nu} \times \mathbf{R}^{+},\left(\phi_{\nu} \times \mathrm{id}\right)\right)$ on $M_{\infty}$

$$
\nabla_{i_{1}} \cdots \nabla_{i_{l}} T_{j_{1} \cdots j_{q}}^{m_{1} \cdots m_{r}}=\frac{\partial^{l} T_{j_{1} \cdots j_{q}}^{m_{1} \cdots m_{r}}}{\partial X_{i_{1}} \cdots \partial X_{i_{l}}}+L(T)
$$


with $L=\sum_{|\alpha|<l} L_{\alpha}(\omega, z) \partial^{\alpha}$ and $L_{\alpha}$ an $(l+k) n \times k n$ matrix. Moreover there exist translation invariant matrices $L_{\alpha}^{\infty}(\omega)$ such that for all $\beta \in \mathbf{N}^{n}$

$$
\lim _{z \rightarrow \infty} \partial^{\beta}\left(L_{\alpha}(\omega, z)-L_{\alpha}^{\infty}(\omega)\right)=0
$$

uniformly on compact subsets of $\Omega_{\nu}$.

Proof. This is a straightforward calculation that uses condition (2.3.1).

3. Weighted Sobolev spaces. As was mentioned in the introduction weighted Sobolev spaces need to be used on noncompact manifolds in order to get Fredholm results for elliptic operators. Moreover which weights should be used depends on the operator. In the case of elliptic operators that are asymptotically translation invariant, the proper weights are the functions $\exp (\tilde{\delta})$ with $\tilde{\delta} \in C^{\infty}(M)$ and $\tilde{\delta} / z$ locally constant on $M_{\infty}$. Since $\tilde{\delta} / z$ is locally constant on $M_{\infty}$ and since we do not care what $\tilde{\delta}$ is on $M_{0}$, we may identify it with an element, $\delta$, of $\mathbf{R}^{L}$, where $L$ is the number of ends of $M$.

Definition (3.1). If $L=$ number of components of $\partial M_{0}$ and $\delta \in \mathbf{R}^{L}$, then $\delta z$ is a $C^{\infty}$ function on $M$ that on the $l$ th component of $M_{\infty}$ equals $\delta_{l} z$.

Notation (3.2). If $\delta_{1}, \delta_{2} \in \mathbf{R}^{L}$, then $\delta_{1} \leqslant \delta_{2}$ means $\delta_{1 l} \leqslant \delta_{2 l}$ for $l=1, \ldots, L$.

With this notation we are ready to define the weighted Sobolev spaces needed for Laplacians associated with asymptotically translation invariant metrics. In what follows we let $E$ be either $T_{r}^{q}(M)$ or $\wedge^{q}(M)$ and, as usual, take $h$ to be asymptotically translation invariant.

Definition (3.3). Let $1<p<\infty, \delta \in \mathbf{R}^{L}$, and $s \in \mathbf{N}$. For $\sigma \in L_{s \text {,loc }}^{p}(E)$ set

$$
\|\boldsymbol{\sigma}\|_{p, s, \delta}=\left(\sum_{t=0}^{s} \int_{M}\left\|e^{\delta z} D_{(h)}^{t} \sigma\right\|_{h}^{p} d V_{h}\right)^{1 / p} .
$$

Definition (3.4). If $1<p<\infty, \delta \in \mathbf{R}^{L}$, and $s \in \mathbf{N}$, then

$$
W_{s, \delta}^{p}(E)=\left\{\sigma \in L_{s, \mathrm{loc}}^{p}(E) \mid\|\sigma\|_{p, s, \delta}<\infty\right\} .
$$

If $1<p<\infty, \delta \in \mathbf{R}^{L}$, and $-s \in \mathbf{N}$ then $W_{s, \delta}^{p}(E)$ is the dual space of $W_{-s,-\delta}^{p^{\prime}}(E)$, where $1 / p+1 / p^{\prime}=1$.

A few remarks about these definitions are in order. First of all, it is easy to show that these are Banach spaces. Secondly notice that by picking $\delta \in \mathbf{R}^{L}$ instead of in $\mathbf{R}$ we allow for different growth on the different ends of $M$. Thirdly note that the standard Sobolev space, $L_{s}^{p}(E, h)$, equals $W_{s, 0}^{p}(E)$ and that if $\delta_{1}, \delta_{2} \in \mathbf{R}^{L}$ with $\delta_{1} \leqslant \delta_{2}$ and $s \geqslant 0$, then $W_{s, \delta_{2}}^{p}(E) \subset W_{s, \delta_{1}}^{p}(E)$. Thus we have embedded $L_{s}^{p}(E, h)$ into a family of weighted Sobolev spaces. Finally observe that any two asymptotically translation invariant metrics give rise to equivalent weighted Sobolev norms. Indeed it follows from Lemma (2.10) that the norm (3.3.1) is equivalent to the following metric independent one.

Definition (3.5). Let $\left\{\left(\Omega_{\nu}, \tilde{\phi}_{\nu}\right)\right\}_{\nu=1}^{N}$ be an atlas for $\partial M_{0}$ and set $M_{\nu}=\Omega_{\nu} \times(0, \infty)$ and $\phi_{\nu}=\left(\tilde{\phi}_{\nu}\right.$, id $)$. Then $\left\{\left(M_{\nu}, \phi_{\nu}\right)\right\}_{\nu=1}^{N}$ is an atlas for $M_{\infty}$. Extend this to a finite atlas, $\left\{\left(M_{\nu}, \phi_{\nu}\right)\right\}_{\nu=1}^{N+J}$ of $M$ and let $\left\{\psi_{\nu}\right\}_{\nu=1}^{N+J}$ be a subordinate partition of unity. If 
$1<p<\infty, \delta \in \mathbf{R}^{L}, s \in \mathbf{N}$, and $\sigma \in L_{s, \text { loc }}^{p}(E)$, then

$$
\|\sigma\|_{\tilde{p}, s, \delta}^{\tilde{\delta}}=\left(\sum_{\nu=1}^{N+J} \sum_{\substack{|\alpha|=0 \\ \mid \alpha}}^{s} \sum_{\substack{i_{1}, \ldots, i_{q}=1 \\ j_{1}, \ldots, j_{r}=1}}^{n} \int_{\phi_{\nu}\left(M_{\nu}\right)}\left|\exp \left(\tilde{\delta} \circ \phi_{\nu}^{-1}\right) \partial^{\alpha}\left(\psi_{\nu} \sigma_{i_{1} \cdots i_{q}}^{j_{1} \cdots j_{r}}\right) \circ \phi_{\nu}^{-1}\right|^{p} d x\right)^{1 / p} .
$$

It is the norm (3.5.1) that is used in [10] and so the Fredholm results there hold for the spaces in (3.4). Before stating these results we must say what an asymptotically translation invariant operator is. Recall that if $A: C^{\infty}(E) \rightarrow C^{\infty}(E)$ is an $m$ th order linear differential operator, then in terms of the covariant derivative of $h$ we have $A=\sum_{t=0}^{m} a_{t} \cdot D_{(h)}^{t}$ with $a_{t} \in C^{\infty}\left(T_{q+r+t}^{q+r} M\right)$ and "." meaning tensor product followed by contraction. A differential operator is said to be translation invariant if it is invariant under the $\mathbf{R}^{+}$-action on $M_{\infty}$.

Definition (3.6). Suppose that $A=\sum_{t=0}^{m} a_{t} \cdot D_{(h)}^{t}$ and $A_{\infty}=\sum_{t=0}^{m} a_{t}^{\infty} \cdot D_{(h)}^{t}$ are two $m$ th order differential operators and that $A_{\infty}$ is translation invariant. The operator $A$ is asymptotic to $A_{\infty}$, written $A \sim A_{\infty}$, if for every $l \in \mathbf{N}$ and $0 \leqslant t \leqslant m$

$$
\lim _{z \rightarrow \infty} \sup _{\partial M_{0}}\left\|D_{(h)}^{l}\left(a_{t}-a_{t}^{\infty}\right)\right\|_{h}=0
$$

If $A \sim A_{\infty}$ then $A$ is said to be asymptotically translation invariant.

It is easy to see from Lemma (2.10) that this definition is independent of the choice of asymptotically translation invariant metric, $h$, used to express $A$ and $A_{\infty}$.

The Fredholm results we need are the following.

Theorem (3.7). Suppose $A, A_{\infty}: C^{\infty}(E) \rightarrow C^{\infty}(E)$ are mth order, elliptic differential operators, $A_{\infty}$ is translation invariant, and $A \sim A_{\infty}$. Then

(3.7.1) $A$ is continuous from $W_{s+m, \delta}^{p}(E)$ to $W_{s, \delta}^{p}(E)$ for all $1<p<\infty, s \in \mathbf{Z}$, and $\delta \in \mathbf{R}^{L}$.

(3.7.2) If $1<p<\infty, s \in \mathbf{Z}$, and $\delta \in \mathbf{R}^{L}$, then for all $\boldsymbol{\sigma} \in L_{s+m \text {,loc }}^{p}(E)$

$$
\|\boldsymbol{\sigma}\|_{p, s+m, \delta} \leqslant c\left(\|A \boldsymbol{\sigma}\|_{p, s, \delta}+\|\sigma\|_{p, s, \delta}\right)
$$

for some $c$ independent of $\boldsymbol{\sigma}$.

(3.7.3) There is a subset $\mathscr{D}_{A} \subset \mathbf{R}^{L}$ such that if $1<p<\infty$ and $s \in \mathbf{N}$, then $A$ : $W_{s+m, \delta}^{p}(E) \rightarrow W_{s, \delta}^{p}(E)$ is Fredholm if and only if $\delta \in \mathbf{R}^{L}-\mathscr{D}_{A}$.

Proof. See inequality (2.4) in [10] for (3.7.2) and $\S 7$ in [10] for (3.7.3).

Much can be said about the set $\mathscr{D}_{A}$. For instance $\mathscr{D}_{A}=\mathscr{D}_{A_{\infty}}$. Also it is of measure zero in $\mathbf{R}^{L}$. In fact it is of the form

$$
\mathscr{D}_{A}=\left(\mathscr{D}_{A_{1}} \times \mathbf{R}^{L-1}\right) \cup\left(\mathbf{R} \times \mathscr{D}_{A_{2}} \times \mathbf{R}^{L-2}\right) \cup \cdots \cup\left(\mathbf{R}^{L-1} \times \mathscr{D}_{A_{L}}\right)
$$

with each $\mathscr{D}_{A_{i}}$ a countable discrete set in $\mathbf{R}$. That this is so comes from the fact that $\left(\delta_{1}, \ldots, \delta_{L}\right)$ is in $\mathscr{D}_{A}$ if at least one of the $\delta_{l}$ is an eigenvalue of an associated generalized eigenvalue problem for $A_{\infty}$ on $\partial M_{0} \times \mathbf{R}$. (See [10] for details.) For example, suppose $h$ is a metric that is the product metric $d z^{2}+h_{0}$ on $M_{\infty}$. Then on $M_{\infty}$ a $q$-form $\sigma$ equals $\psi(\omega, z)+\tau(\omega, z) \wedge d z$ for some $\psi \in \Gamma \wedge^{q}\left(\partial M_{0}\right)$ and $\tau \in$ $\Gamma \wedge^{q-1}\left(\partial M_{0}\right)$ and

$$
\Delta_{h} \sigma=-\frac{\partial^{2} \psi}{\partial z^{2}}+\Delta_{h_{0}} \psi+(-1)^{q+1}\left(-\frac{\partial^{2} \tau}{\partial z^{2}}+\Delta_{h_{0}} \tau\right) \wedge d z
$$


In this case $\delta=\left(\delta_{1}, \ldots, \delta_{L}\right)$ is in $\mathscr{D}_{\Delta_{h}}$ if for some $l$ we have that $\delta_{l}$ is an eigenvalue for either $\Delta_{h_{0}}: C^{\infty}\left(\wedge^{q} \Omega_{l}\right) \rightarrow C^{\infty}\left(\wedge^{q} \Omega_{l}\right)$ or $\Delta_{h_{0}}: C^{\infty}\left(\wedge^{q-1} \Omega_{l}\right) \rightarrow C^{\infty}\left(\wedge^{q-1} \Omega_{l}\right)$ where $\Omega_{l}$ is the $l$ th component of $\partial M_{0}$. Thus for instance

$$
\Delta_{h}: L_{2}^{p}\left(\wedge^{q} M\right)=W_{2,0}^{p}\left(\bigwedge^{q} M\right) \rightarrow L^{p}\left(\wedge^{q} M\right)=W_{0,0}^{p}\left(\bigwedge^{q} M\right)
$$

is Fredholm if and only if $H_{D R}^{q-1}\left(\partial M_{0}\right)=H_{D R}^{q}\left(\partial M_{0}\right)=0$. Finally let us point out that even though $\mathscr{D}_{A}=\mathscr{D}_{A_{\infty}}$ it is not the case that ind $\delta=$ ind $_{\delta} A_{\infty}$ if $\delta \in \mathbf{R}^{L}-\mathscr{D}_{A}$. However, if $\delta_{1}<\delta_{2}$ and $\delta_{1}, \delta_{2} \in \mathbf{R}^{L}-\mathscr{D}_{A}$, then it is true that ind $\delta_{1} A-\operatorname{ind}_{\delta_{2}} A=$ ind $_{\delta_{1}} A_{\infty}-$ ind $_{\delta_{2}} A_{\infty}$. (For details see $[10]$.)

The reader may feel that the Fredholm result follows from the a priori inequality (3.7.2). However since $M$ is not compact the imbedding $W_{s+m, \delta}^{p}(E) \rightarrow W_{s, \delta}^{p}(E)$ is not compact and so (3.7.2) does not imply Fredholm. We conclude this section by presenting various properties of the spaces $W_{s, \delta}^{p}(E)$, including a weighted compact imbedding theorem.

Proposition (3.9). $C_{0}^{\infty}(E)$ is dense in $W_{s, \delta}^{p}(E)$.

Proof. This follows from the fact that (3.5.1) gives an equivalent norm for these spaces and classical facts about Sobolev spaces in $\mathbf{R}^{n}$.

Theorem (3.10) (Weighted Sobolev Embedding). There is a continuous embed$\operatorname{ding} W_{s, \delta}^{p}(E) \rightarrow W_{\bar{s}, \bar{\delta}}^{\bar{p}_{\bar{\delta}}}(E)$ if

(i) $s-\bar{s} \geqslant n / p-n / \bar{p}(n=\operatorname{dim} M)$,

(ii) $s \geqslant \bar{s} \geqslant 0$ and either

(iii) $1<p \leqslant \bar{p}<\infty$ with $\bar{\delta} \leqslant \delta$ or

(iii') $1<\bar{p}<p<\infty$ with $\bar{\delta}<\delta$.

Proof. See Lemma 7.2 in [10].

In proving the compact embedding theorem we need the following

Lemma (3.11). For $\mathscr{F} \subset M$, a measurable set, define

$$
\|\sigma\|_{p, s, \delta}(\mathscr{F})=\left(\sum_{t=0}^{s} \int_{\mathscr{F}}\left\|e^{\delta z} D_{(h)}^{t} \sigma\right\|_{h}^{p} d V_{h}\right)^{1 / p} .
$$

Also let $M_{R}=M_{0} \cup\left(\partial M_{0} \times(0, R)\right)$. If $s, \bar{s}, p, \bar{p}, \delta$ and $\bar{\delta}$ satisfy the conditions in (3.10), $\sigma \in W_{s, \delta}^{p}(E)$, and $R>1$, then

$$
\|\sigma\|_{\bar{p}, \bar{s}, \bar{\delta}}\left(M-M_{2 R}\right) \leqslant c\|\sigma\|_{p, s, \delta}\left(M-M_{R}\right)
$$

for some $c$ independent of $\sigma$ and $R$.

Proof. For $\tilde{\phi} \in C_{0}^{\infty}([0, \infty))$ such that $\tilde{\phi} \equiv 1$ on $[0,1]$ and $\tilde{\phi} \equiv 0$ on $[2, \infty)$, take $\tilde{\phi}_{R}(z)=\tilde{\phi}(z / R)$. Define $\phi_{R} \in C_{0}^{\infty}(M)$ by

$$
\phi_{R}(x)= \begin{cases}1 & \text { if } x \in M_{0}, \\ \tilde{\phi}_{R}(z) & \text { if } x=(\omega, z) \in M_{\infty} .\end{cases}
$$

Clearly we have

$$
\|\boldsymbol{\sigma}\|_{\bar{p}, \bar{s}, \bar{\delta}}\left(M-M_{2 R}\right) \leqslant\left\|\left(1-\phi_{R}\right) \boldsymbol{\sigma}\right\|_{\bar{p}, \bar{s}, \bar{\delta}} .
$$


By (3.10) we have

$$
\left\|\left(1-\phi_{R}\right) \sigma\right\|_{\bar{p}, \bar{s}, \bar{\delta}} \leqslant c\left\|\left(1-\phi_{R}\right) \sigma\right\|_{p, s, \delta}=c\left\|\left(1-\phi_{R}\right) \sigma\right\|_{p, s, \delta}\left(M-M_{R}\right) .
$$

However from the construction of $\phi_{R}$ we see that if $R>1$, then

$$
\left\|\left(1-\phi_{R}\right) \sigma\right\|_{p, s, \delta}\left(M-M_{R}\right) \leqslant c\|\sigma\|_{p, s, \delta}\left(M-M_{R}\right)
$$

with $c$ independent of $\sigma$ and $R$.

Theorem (3.12) (COMPACT EMBEDding). If

(i) $s-\bar{s}>n / p-n / \bar{p}$,

(ii) $s>\bar{s} \geqslant 0$, and

(iii) $\bar{\delta}<\delta$,

then the embedding $W_{s, \delta}^{p}(E) \rightarrow W_{\bar{s}, \bar{\delta}}^{\bar{p}}(E)$ is compact.

Proof. Suppose $\left\{\sigma_{j}\right\}$ is a bounded sequence in $W_{s, \delta}^{p}(E)$. Using the notation in (3.11) and Minkowski's inequality we get

$$
\left\|\sigma_{j}\right\|_{\bar{p}, \bar{s}, \bar{\delta}} \leqslant c\left(\left\|\sigma_{j}\right\|_{\bar{p}, \bar{s}, \bar{\delta}}\left(M_{2 R}\right)+\left\|\sigma_{j}\right\|_{\bar{p}, \bar{s}, \bar{\delta}}\left(M-M_{2 R}\right)\right) .
$$

Let $\hat{\delta}$ be defined by $\hat{\delta}_{l}=\left(\delta_{l}+\bar{\delta}_{l}\right) / 2$. Then $\bar{\delta}<\hat{\delta}<\delta$ and so by (3.11.2)

$$
\left\|\sigma_{j}\right\|_{\bar{p}, \bar{s}, \bar{\delta}}\left(M-M_{2 R}\right) \leqslant c\left\|\sigma_{j}\right\|_{p, s, \hat{\delta}}\left(M-M_{R}\right) \text {. }
$$

For all $j$ we have

$$
\begin{aligned}
\left\|\sigma_{j}\right\|_{p, s, \hat{\delta}}\left(M-M_{R}\right) & =\left(\sum_{t=0}^{s} \int_{\partial M_{0} \times(R, \infty)}\left\|e^{(\hat{\delta}-\delta) z} e^{\delta z} D_{(h)}^{t} \sigma_{j}\right\|_{h}^{p} d V_{h}\right)^{1 / p} \\
& \leqslant e^{-b R}\left\|\sigma_{j}\right\|_{p, s, \delta} \leqslant c e^{-b R}
\end{aligned}
$$

with $0<b=\min \left\{\delta_{l}-\hat{\delta}_{l}\right\}$ and $c=\sup _{j}\left\{\left\|\sigma_{j}\right\|_{p, s, \delta}\right\}$. On the other hand, it follows from the classical Rellich theorem that there is a subsequence of $\left\{\sigma_{j}\right\}$ that converges in the \|\|$_{\bar{p}, \bar{s}, \bar{\delta}}\left(M_{2 R}\right)$ norm. Thus if $N \in \mathbf{N}$, we may pick $R$ large enough so that there is a subsequence, $\left\{\tilde{\sigma}_{j}\right\}$ of $\left\{\sigma_{j}\right\}$ that satisfies

$$
\left\|\tilde{\sigma}_{j}-\tilde{\sigma}_{m}\right\|_{\bar{p}, \bar{s}, \bar{\delta}} \leqslant 2^{-N}
$$

for all $j$ and $m$.

Let $\left\{\sigma_{j 1}\right\}$ be a subsequence of $\left\{\sigma_{j}\right\}$ such that $\left\|\sigma_{j 1}-\sigma_{m 1}\right\|_{\bar{p}, \bar{s}, \bar{\delta}} \leqslant 2^{-1}$ for all $j$ and $m$. For $N=2,3, \ldots$ pick $\left\{\sigma_{j N}\right\}$ to be a subsequence of $\left\{\sigma_{j N-1}\right\}$ satisfying $\left\|\sigma_{j N}-\sigma_{m N}\right\|_{\bar{p}, \bar{s}, \bar{\delta}} \leqslant 2^{-N}$ for all $j$ and $m$. Finally let $\hat{\sigma}_{j}=\sigma_{j j}$. Since $\left\|\hat{\sigma}_{j}-\hat{\sigma}_{m}\right\|_{\bar{p}, \bar{s}, \bar{\delta}} \leqslant$ $2^{-K}$ for $j, m>K$, the sequence $\left\{\hat{\boldsymbol{\sigma}}_{j}\right\}$ converges in $W_{\bar{s}, \bar{\delta}}^{p}(E)$.

4. Weighted Sobolev spaces for admissible metrics. For the Laplacians associated with a general admissible metric, $g=e^{2 \rho} h$, the Sobolev spaces introduced in the last section are not the right ones to use, unless $\rho$ is bounded. In this section we introduce the correct spaces and give some of their properties. As before $E$ is either $T_{r}^{q} M$ or $\wedge^{q} M$ and $h$ is an asymptotically translation invariant metric.

Definition (4.1). Let $1<p<\infty, \delta \in \mathbf{R}^{L}, a \in \mathbf{R}$, and $s \in \mathbf{N}$. Suppose $g=e^{2 \rho} h$ is admissible. If $\sigma \in L_{s, \text { loc }}^{p}(E)$, then

$$
\|\boldsymbol{\sigma}\|\left(W_{s, \delta, a}^{p}(E, g)\right)=\left(\sum_{t=0}^{s} \int_{M}\left\|e^{\delta z+(t+a) \rho} D_{(g)}^{t} \boldsymbol{\sigma}\right\|_{g}^{p} d V_{g}\right)^{1 / p} .
$$


Definition (4.2). If $1<p<\infty, \delta \in \mathbf{R}^{L}, a \in \mathbf{R}$, and $s \in \mathbf{N}$, then

$$
W_{s, \delta, a}^{p}(E, g)=\left\{\sigma \in L_{s, \mathrm{loc}}^{p}(E) \mid\|\boldsymbol{\sigma}\|\left(W_{s, \delta, a}^{p}(E, g)\right)<\infty\right\} \text {. }
$$

If $1<p<\infty, \delta \in \mathbf{R}^{L}, a \in \mathbf{R}$, and $-s \in \mathbf{N}$, then $W_{s, \delta, a}^{p}(E, g)$ is the dual space of $W_{-s,-\delta,-a}^{p^{\prime}}(E, g)$, where $1 / p+1 / p^{\prime}=1$.

Once again it is straightforward to show that these are Banach spaces. Also notice that if $\rho$ is bounded, and so $g$ is asymptotically translation invariant, then $W_{s, \delta, a}^{p}(E, g)=W_{s, \delta}^{p}(E)$, equipped with an equivalent norm. Note too that $W_{0,0,0}^{p}(E, g)=L^{p}(E, g)$. However, if $\rho$ is not bounded, then $W_{s, 0,0}^{p}(E, g)$ is not the classical Sobolev space, $L_{s}^{p}(E, g)$.

The need for the term $\exp ((t+a) \rho)$ in (4.1.1) may seem strange. It is best explained by Proposition (4.4). Before giving that proposition let us mention the following useful representation for elements of $W_{s, \delta, a}^{p}\left(T_{r}^{q} M, g\right)$ for $s<0$.

Proposition (4.3). For every $\sigma \in W_{s, \delta, a}^{p}\left(T_{r}^{q} M, g\right)$ with $s<0$, there is a unique $(1-s)$-tuple, $\left(\sigma_{0}, \ldots, \sigma_{-s}\right) \in \bigoplus_{t=0}^{-s} W_{0, \delta, a-t}^{p}\left(T_{r}^{q+t} M, g\right)$, such that

$$
\sigma[\phi]=\int_{M} \sum_{t=0}^{-s}\left\langle\sigma_{t}, D_{(g)}^{t} \phi\right\rangle_{g} d V_{g}
$$

for $\phi \in W_{-s,-\delta,-a}^{p^{\prime}}\left(T_{r}^{q} M, g\right)$, and

$$
\|\boldsymbol{\sigma}\|\left(W_{s, \delta, a}^{p}\left(T_{r}^{q} M, g\right)\right)=\left(\sum_{t=0}^{-s}\left[\left\|\sigma_{t}\right\|\left(W_{0, \delta, a-t}^{p}\left(T_{r}^{q+t} M, g\right)\right)\right]^{p}\right)^{1 / p} .
$$

PROOF. The standard proof, as in [1], can be used here too.

Proposition AND Definition (4.4). For $g=e^{2 \rho} h$ admissible define the operator $K_{a, p}$ on $W_{s, \delta, a}^{p}\left(T_{r}^{q} M, g\right)$ as follows: if $s \in \mathbf{N}$, then

$$
K_{a, p} \boldsymbol{\sigma}=e^{(a+r-q+n / p) \rho} \boldsymbol{\sigma} .
$$

If $-s \in \mathbf{N}$ and

$$
\left(\sigma_{0}, \ldots, \sigma_{-s}\right) \in \bigoplus_{t=0}^{-s} W_{0, \delta, a-t}^{p}\left(T_{r}^{q+t} M, g\right)
$$

represents $\sigma \in W_{s, \delta, a}^{p}\left(T_{r}^{q} M, g\right)$ as in (4.3), then

$$
\left(K_{a, p} \sigma\right)[\psi]=\int_{M} \sum_{t=0}^{-s}\left\langle\exp \left(\left(a+r-q-t+\frac{n}{p}\right) \rho\right) \sigma_{t}, D_{(h)}^{t} \phi\right\rangle_{h} d V_{h}
$$

for $\phi \in W_{-s,-\delta, 0}^{p^{\prime}}\left(T_{r}^{q} M, h\right)$.

For all $s \in \mathbf{Z}$

$$
K_{a, p}: W_{s, \delta, a}^{p}\left(T_{r}^{q} M, g\right) \rightarrow W_{s, \delta, 0}^{p}\left(T_{r}^{q} M, h\right)=W_{s, \delta}^{p}\left(T_{r}^{q} M\right)
$$

is a Banach space isomorphism onto.

Proof. If $s \geqslant 0$, then

$$
\begin{aligned}
& \left\|e^{(a+r-q+n / p) \rho} \sigma\right\|_{p, s, \delta} \\
& \leqslant\left(\sum_{t=0}^{s} \sum_{b \leqslant t} c_{b t} \int_{M}\left\|e^{\delta z+(a+r-q+n / p) \rho} D_{(h)}^{t-b} \rho D_{(h)}^{b} \sigma\right\|_{h}^{p} d V_{h}\right)^{1 / p} .
\end{aligned}
$$


It follows from condition (2.3.1) on $\rho$ that this is

$$
\begin{aligned}
& \leqslant c\left(\sum_{b=0}^{s} \int_{M}\left\|e^{\delta z+(a-q+r+n / p) \rho} D_{(h)}^{b} \sigma\right\|_{h}^{p} d V_{h}\right)^{1 / p} \\
& =c\left(\sum_{b=0}^{s} \int_{M}\left\|e^{\delta z+(a+b) \rho} D_{(h)}^{b} \sigma\right\|_{g}^{p} d V_{g}\right)^{1 / p} .
\end{aligned}
$$

Using Lemma (2.10) we get that this in turn is $\leqslant c\|\sigma\|\left(W_{s, \delta, a}^{p}\left(T_{r}^{q} M, g\right)\right)$.

Thus (4.4.3) is continuous for $s \in \mathbf{N}$. In exactly the same way we can show that $\left(K_{a, p}\right)^{-1}$ defined by

$$
\left(K_{a, p}\right)^{-1} \gamma=e^{(q-r-a-n / p) \rho} \gamma
$$

is continuous. This proves the proposition for $s \geqslant 0$. The case of $s<0$ follows easily from this.

We shall also use $K_{a, p}$ on $W_{s, \delta, a}^{p}\left(\wedge^{q} M, g\right)$ by considering $\wedge^{q} M$ to be in $T_{0}^{q} M$. The proposition is true then in this case too.

Corollary (4.5). If $g$ is admissible, then $C_{0}^{\infty}(0)$ is dense in $W_{s, \delta, a}^{p}(E, g)$.

Proof. The operator $K_{a, p}$ obviously preserves supports and so this follows from Proposition (3.9).

Proposition (4.6). For admissible $g$

$$
D_{(g)}: W_{s, \delta, a}^{p}(E, g) \rightarrow W_{s-1, \delta, a+1}^{p}\left(T_{r}^{q+1} M, g\right)
$$

is continuous.

Proof. For $s \geqslant 1$ this follows easily from Definition (4.1). For $s<1$ we have by the density of $C_{0}^{\infty}\left(T_{r}^{q+1}\right)$ in $W_{1-s,-\delta,-a-1}^{p^{\prime}}\left(T_{r}^{q+1} M, g\right)$ that

$$
\left(D_{(g)} \boldsymbol{\sigma}\right)[\psi]=\sigma\left[D_{(g)}^{*} \psi\right]
$$

where $\psi \in W_{1-s,-\delta,-a-1}^{p^{\prime}}\left(T_{r}^{q+1} M, g\right)$ and $D_{(g)}^{*}$ is the formal adjoint of $D_{(g)}$. Thus (4.6.1) is continuous for $s \leqslant 0$ provided

$$
D_{(g)}^{*}: W_{1-s,-\delta,-a-1}^{p^{\prime}}\left(T_{r}^{q+1} M, g\right) \rightarrow W_{-s,-\delta,-a}^{p^{\prime}}\left(T_{r}^{q} M, g\right)
$$

is continuous. But (4.6.3) is continuous because

$$
\left(D_{(g)}^{*} \psi\right)_{i_{1} \cdots i_{q}}^{j_{1} \cdots j_{r}}=e^{-2 \rho} h^{l k} \nabla, \psi_{k_{1} \cdots i_{1} \cdots i_{q}}^{j_{1} \cdots j_{r}}
$$

with $\nabla$ the covariant derivative for $g$.

It is apparent from the proof of (4.4) that $K_{a, p}$ would not be bounded were the term $e^{t \rho}$ not in the norm (4.1.1). However once one has introduced $e^{t \rho}$ then $e^{a \rho}$ is needed too in order for Proposition (4.6) to be true.

An immediate corollary of (4.6) is

Corollary (4.7). For $g$ admissible, $s \in \mathbf{Z}, \delta \in \mathbf{R}^{L}, a \in \mathbf{R}$ and $1<p<\infty$ the following operators are continuous:

$$
\begin{gathered}
d: W_{s, \delta, a}^{p}\left(\bigwedge^{q} M, g\right) \rightarrow W_{s-1, \delta, a+1}^{p}\left(\bigwedge^{q+1} M, g\right), \\
d_{g}^{*}: W_{s, \delta, a}^{p}\left(\bigwedge^{q} M, g\right) \rightarrow W_{s-1, \delta, a+1}^{p}\left(\bigwedge^{q-1} M, g\right),
\end{gathered}
$$




$$
\begin{gathered}
\Delta_{g}: W_{s, \delta, a}^{p}\left(\bigwedge^{q} M, g\right) \rightarrow W_{s-2, \delta, a+2}^{p}\left(\wedge^{q} M, g\right), \\
d+d_{g}^{*}: \bigoplus_{q=0}^{n} W_{s, \delta, a}^{p}\left(\bigwedge^{q} M, g\right) \rightarrow \bigoplus_{q=0}^{n} W_{s-1, \delta, a+1}^{p}\left(\bigwedge^{q} M, g\right) .
\end{gathered}
$$

Proposition (4.4) allows us to carry over the properties of the spaces $W_{s, \delta}^{p}(E)$ in $\S 3$ to the spaces $W_{s, \delta, a}^{p}(E, g)$.

Theorem (4.8) (Weighted Sobolev Embedding). Suppose g is admissible. There is a continuous embedding

$$
W_{s, \delta, a}^{p}(E, g) \rightarrow W_{\bar{s}, \bar{\delta}, a+n\left(1 / p-1 / p^{\prime}\right)}^{\overline{\bar{\delta}}}(E, g)
$$

if

(i) $s-\bar{s} \geqslant n(1 / p-1 / \bar{p})$

(ii) $s \geqslant \bar{s} \geqslant 0$ and either

(iii) $1<p \leqslant \bar{p}<\infty$ with $\bar{\delta} \leqslant \delta$ or

(iii') $1<\bar{p}<p<\infty$ with $\bar{\delta}<\delta$.

Proof. Let $g=e^{2 \rho} h$. By (4.4) and (3.10) we have the sequence of continuous maps:

$$
\begin{aligned}
& W_{s, \delta, a}^{p}(E, g) \stackrel{K_{a, p}}{\rightrightarrows} W_{s, \delta}^{p}(E) \rightarrow W_{\bar{s}, \bar{\delta}}^{\bar{p}}(E)
\end{aligned}
$$

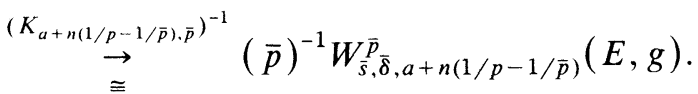

Furthermore by (4.4.1) and (4.4.6) if $\sigma \in W_{s, \delta, a}^{p}(E, g)$, then in (4.8.2) it is mapped to

$$
e^{(q-r-a-n / p+n / \bar{p}-n / \bar{p}) \rho} \cdot e^{(a+r-q+n / p) \rho} \sigma=\sigma .
$$

THEOREM 4.9 (COMPACT EMBEDDING). The embedding (4.8.1) is compact when

(i) $s-\bar{s}>n / p-n / \bar{p}$,

(ii) $s>\bar{s} \geqslant 0$ and

(iii) $\bar{\delta}<\delta$.

Proof. Theorem (3.11) implies the middle map in (4.8.2) is compact when (i)-(iii) hold.

5. Fredholm results. In this section the Fredholm properties of Laplacians associated with admissible metrics are established. Throughout the section $h$ is, as usual, an asymptotically translation invariant metric. We start with a straightforward lemma.

LeMMA (5.1). If $g=e^{2 \rho} h$ is admissible, then $D_{(g)}^{t}$ is an asymptotically translation invariant operator for every $t \in \mathbf{N}$.

Proof. It is clear from Definition (3.6) that the composition of asymptotically translation invariant operators is again asymptotically translation invariant. Hence we need only consider $t=1$. In that case $D_{(g)}=D_{(h)}+L$ with $L$ a 0 th order operator with coefficients that depend only on $h$ and $D_{(h)} \rho$. The lemma, then, is a consequence of condition (2.3.1) on $\rho$. 
THEOREM (5.2). Let $g=e^{2 \rho} h$ be admissible. For every $1<p<\infty, s \in \mathbf{N}$, and $a \in \mathbf{R}$ there is a subset $\mathscr{D}_{\Delta}=\mathscr{D}_{\Delta}(g, p, q, a)$ of $\mathbf{R}^{L}$ with the properties

$$
\mathscr{D}_{\Delta}=\left(\mathscr{D}_{1} \times \mathbf{R}^{L-1}\right) \cup\left(\mathbf{R} \times \mathscr{D}_{2} \times \mathbf{R}^{L-2}\right) \cup \cdots \cup\left(\mathbf{R}^{L-1} \times \mathscr{D}_{L}\right)
$$

with each $\mathscr{D}$, countable and discrete.

(5.2.2) $\mathscr{D}_{\Delta}$ depends on $p, q, a$, the metric $g$, and $n=\operatorname{dim} M$, but not $s \in \mathbf{N}$.

$$
\Delta_{g}: W_{s+2, \delta, a}^{p}\left(\bigwedge^{q} M, g\right) \rightarrow W_{s, \delta, a+2}^{p}\left(\wedge^{q} M, g\right)
$$

is Fredholm if and only if $\delta \in \mathbf{R}^{L}-\mathscr{D}_{\Delta}$.

Proof. For $b \in \mathbf{R}$, define $A(b)$ to be the operator

$$
A(b)=e^{(2+b) \rho} \Delta_{g} e^{-b \rho} .
$$

Letting $\bar{\nabla}$ be the covariant derivative for $g$, we have by a standard identity that

$$
(A(b) \sigma)_{i_{1} \cdots i_{q}}=e^{(2+b) \rho}\left[e^{-b \rho} \Delta_{g} \sigma_{i_{1} \cdots i_{q}}-2\left(\bar{\nabla}^{k} e^{-b \rho} \bar{\nabla}_{k} \sigma_{i_{1} \cdots i_{q}}\right)-\left(\Delta_{g} e^{-b \rho}\right) \sigma_{i_{1} \cdots i_{q}}\right] .
$$

Computing the second and third terms on the right-hand side of (5.2.5) and using the definition of $\Delta_{g}$ we get

$$
\begin{aligned}
(A(b) \sigma)_{i_{1} \cdots i_{q}}=e^{2 \rho}\left[-\bar{\nabla}^{k} \bar{\nabla}_{k} \sigma_{i_{1} \cdots i_{q}}\right. & +\sum_{\nu=1}^{q}(-1)^{\nu}\left(\bar{\nabla}_{i_{\nu}} \bar{\nabla}^{k}-\bar{\nabla}^{k} \bar{\nabla}_{i_{\nu}}\right) \sigma_{k i_{1} \cdots \hat{i}_{\nu} \cdots i_{q}} \\
& \left.+2 b \bar{\nabla}_{\rho}^{k} \bar{\nabla}_{k} \sigma_{i_{1} \cdots i_{u}}-\left(b^{2}\|\bar{\nabla} \rho\|_{g}^{2}-b \Delta_{g} \rho\right) \sigma_{i_{1} \cdots i_{q}}\right] .
\end{aligned}
$$

Since $g=e^{2 \rho} h$, it follows from (5.2.6) that

$$
\begin{aligned}
& (A(b) \sigma)_{i_{1} \cdots i_{q}} \\
& =h^{t k}\left[-\bar{\nabla}_{t} \bar{\nabla}_{k} \sigma_{i_{1} \cdots i_{q}}+\sum_{\nu=1}^{q}(-1)^{\nu}\left(\bar{\nabla}_{i_{\nu}} \bar{\nabla}_{t}-\bar{\nabla}_{t} \bar{\nabla}_{i_{\nu}}\right) \sigma_{k i_{1} \cdots \hat{i}_{\nu} \cdots i_{q}}+2 b\left(\bar{\nabla}_{t} \rho \bar{\nabla}_{k} \sigma_{i_{1} \cdots i_{q}}\right)\right] \\
& \quad+\left(b \Delta_{h} \rho-b(b-n+2)\|\bar{\nabla} \rho\|_{h}\right) \sigma_{i_{1} \cdots i_{q}} .
\end{aligned}
$$

Thus as a result of Lemma (5.1) and condition (2.3.1) on $\rho$, we see that $A(b)$ is an asymptotically translation invariant operator. Hence by Theorem (3.7) there is a subset $\mathscr{D}_{A(b)}$ of $\mathbf{R}^{L}$ of the form described in (5.2.1) with the property that

$$
A(b): W_{s+2, \delta}^{p}\left(\bigwedge^{q} M\right) \rightarrow W_{s, \delta}^{p}\left(\bigwedge^{q} M\right)
$$

is Fredholm if and only if $\delta \in \mathbf{R}^{L}-\mathscr{D}_{A(b)}$. In particular this is true for $b=a-q+$ $n / p$. However, in this case we also have the commutative diagram:

$$
\begin{array}{ccc}
W_{s+2, \delta}^{p}\left(\bigwedge^{q} M\right) & A(a-q+n / p) & W_{s, \delta}^{p}\left(\bigwedge^{q} M\right) \\
\downarrow\left(K_{a, p}\right)^{-1} & \stackrel{\uparrow}{\rightarrow} K_{a+2, P} \\
W_{s+2, \delta, a}^{p}\left(\bigwedge^{q} M, g\right) & \stackrel{\Delta_{g}}{\rightarrow} & W_{s, \delta, a+2}^{p}\left(\bigwedge^{q} M, g\right)
\end{array}
$$


Because of the fact that $\left(K_{a, p}\right)^{-1}$ and $K_{a+2, p}$ are Banach space isomorphisms, the operator (5.2.3) is Fredholm exactly when (5.2.8) with $b=a-q+n / p$ is Fredholm. In fact we see from (5.2.9) that the horizontal maps in that diagram have isomorphic kernels, ranges, and cokernels. In particular when they are Fredholm, they have the same indices.

Using the ideas in the proof of this theorem, we may establish the next fact about harmonic forms.

Proposition (5.3). If $s \in \mathbf{Z}$ and $\sigma \in W_{s, \delta, a}^{p}\left(\wedge^{q} M, g\right)$ is harmonic, then $\sigma \in$ $W_{t, \delta, a}^{p}\left(\wedge^{q} M, g\right)$ for all $t \in \mathbf{Z}$.

Proof. Letting $A(a-q+n / p)$ be as above, we see from (5.2.9) that $K_{a, p} \sigma \in$ $\operatorname{Ker} A(a-q+n / p) \cap W_{s, \delta}^{p}\left(\wedge^{q} M\right)$. Since $\Delta_{g}$ is formally selfadjoint, elliptic, and has $C^{\infty}$ coefficients, $\sigma$ is in $C^{\infty}\left(\wedge^{q} M\right)$ and so $K_{a, p} \sigma$ is also in $L_{k, \text { loc }}^{p}\left(\wedge^{q} M\right)$ for all $k$. Thus if we use the a priori inequality (3.7.2) for $A=A(a-q+n / p)$ and a standard bootstrap argument, we get that $K_{a, p} \sigma \in W_{t, \delta}^{p}\left(\bigwedge^{q} M\right)$ for all $t$. But this means $\sigma \in W_{t, \delta, a}^{p}\left(\bigwedge^{q} M, g\right)$ for all $t$.

Because of this proposition the next definition is independent of $s$.

Definition (5.4). For $g$ admissible

$$
\mathfrak{S}_{\delta, a}^{p}\left(\wedge^{q} M, g\right)=\left\{\sigma \in W_{s, \delta, a}^{p}\left(\wedge^{q} M, g\right) \mid \Delta_{g} \sigma=0\right\} .
$$

Corollary (5.5). For all $0 \leqslant q \leqslant n, \delta \in \mathbf{R}^{L}$, and $a \in \mathbf{R}$ the space $\mathfrak{S}_{\delta, a}^{p}\left(\bigwedge^{q} M, g\right)$ is finite dimensional for admissible $g$.

Proof. For $\delta \in \mathbf{R}^{L}-\mathscr{D}_{\Delta}$ this is merely a property of $\Delta_{g}$ being Fredholm. For $\delta \in \mathscr{D}_{\Delta}$ it follows from the fact that $\mathscr{D}_{\Delta}$ has the form (5.2.1) that there is a $\bar{\delta} \in \mathbf{R}^{L}-\mathscr{D}_{\Delta}$ such that $\bar{\delta}<\delta$. The assertion is then a result of the obvious fact that $\mathfrak{S}_{\mathcal{E}}^{p}, a\left(\bigwedge^{q} M, g\right) \subset \mathfrak{S}_{\bar{\delta}, a}^{p}\left(\bigwedge^{q} M, g\right)$ for $\bar{\delta}<\delta$.

Corollary (5.6). Suppose $g$ is admissible. For each fixed $\delta \in \mathbf{R}^{L}$, the space of harmonic $\sigma$ satisfying

$$
\sup _{M}\left\|e^{\delta z} \boldsymbol{\sigma}\right\|_{g}<\infty
$$

is finite dimensional.

Proof. If $\sigma$ satisfies (5.6.1), then $\sigma \in W_{0, \bar{\delta}, 0}^{p}\left(\wedge^{q} M, g\right)$ for any $\bar{\delta}$ satisfying $\bar{\delta}_{l}-\delta_{l}$ $<-n / p$ for $l=1, \ldots, L$.

We see from Corollary (5.5) that the kernel of

$$
\Delta_{g}: W_{s+2, \delta, a}^{p}\left(\bigwedge^{q} M, g\right) \rightarrow W_{s, \delta, a+2}^{p}\left(\bigwedge^{q} M, g\right)
$$

is always finite dimensional. The kernel of the adjoint of (5.7) is also always finite dimensional; for it is $\mathfrak{S}_{-\delta,-a-2}^{p^{\prime}}\left(\bigwedge^{q} M, g\right)$, where $1 / p+1 / p^{\prime}=1$. Hence if (5.7) is not Fredholm, it is because it does not have a closed range. Nevertheless we shall see in Theorem (5.10) that there is always a Banach space $\tilde{W}_{s+2, \delta, a}^{p}\left(\wedge^{q} M, g\right)$ such that

$$
\Delta_{g}: \tilde{W}_{s+2, \delta, a}^{p}\left(\bigwedge^{q} M, g\right) \rightarrow W_{s, \delta, a+2}^{p}\left(\bigwedge^{q} M, g\right)
$$

is Fredholm. The key is the next technical lemma. 
Lemma (5.9). Let $B_{0}, B_{1}, R_{0}$, and $R_{1}$ be Banach spaces. Suppose $B_{0} \subset B_{1}$ and $R_{0} \subset R_{1}$ as sets and the inclusions are continuous. Also suppose $A$ is a linear operator that is continuous from $B_{i}$ to $R_{i}$ for $i=0,1$. If $A: B_{1} \rightarrow R_{1}$ is Fredholm and $A^{*}$ : $R_{0}^{*} \rightarrow B_{0}^{*}$ has a finite dimensional kernel, then there is a Banach space, $\tilde{B}$, with the properties:

$$
\begin{gathered}
B_{0} \subset \tilde{B} \subset B_{1} \text { and the inclusions are continuous, } \\
\qquad B_{0} \text { is dense in } \tilde{B},
\end{gathered}
$$

$A: \tilde{B} \rightarrow R_{0}$ is defined, continuous, and Fredholm,

Proof. Let $B=\left\{u \in B_{1} \mid A u \in R_{0}\right\}$ and equip $B$ with the norm $\|u\|_{B}=\|u\|_{B_{1}}+$ $\|A u\|_{R_{0}}$. It is straightforward to show that $B$ is a Banach space, that $B_{0} \subset B \subset B_{1}$, as sets, that the inclusions are continuous, and that $A: B \rightarrow R_{0}$ is continuous. In fact $A: B \rightarrow R_{0}$ is Fredholm.

To see this let $K_{B}$ be the kernel of $A$ in $B$ and $W$ be the closure of $A(B)$ in $R_{0}$. Since $B \subset B_{1}$ and $A: B_{1} \rightarrow R_{1}$ is Fredholm, $K_{B}$ is finite dimensional. Also if $w \in W$ then $w \in R_{1}$ and in fact $W$ is in the closure of $A\left(B_{1}\right)$ in $R_{1}$. Thus there is a $u \in B_{1}$ such that $A u=w$. However $u \in B_{1}$ and $A u \in R_{0}$ means $u \in B$. Hence $A(B)=W$. Finally $W$ contains the closure of $A\left(B_{0}\right)$ in $R_{0}$ and so $W$ is finite codimensional, for $A^{*}: R_{0}^{*} \rightarrow B_{0}^{*}$ has a finite dimensional kernel.

Unfortunately, $B$ might not satisfy (5.9.2) and (5.9.4). For this reason we take $\tilde{B}$ to be the closure of $B_{0}$ in $B$ with respect to \|\|$_{B}$ and equip it with the norm \|\|$_{B}$. Clearly $A: \tilde{B} \rightarrow R_{0}$ is continuous and has a finite dimensional kernel. Also, since $A\left(B_{0}\right) \subset A(\tilde{B})$, the closure of $A(\tilde{B})$ in $R_{0}$ is finite codimensional. Thus to finish we only have to show that $A(\tilde{B})$ is closed in $R_{0}$.

Recalling that $K_{B}$ is the kernel of $A$ in $B$, we have from the finite dimensionality of $K_{B}$ that $E=K_{B}+\tilde{B}$ is closed in $B$. Hence $C_{B} E$, the set complement of $E$ in $B$, is open. It follows from the fact that $A: B \rightarrow A(B)$ is an open, onto map that $A\left(C_{B} E\right)$ is open in $A(B)$ and that $A(B)=A(E) \cup A\left(C_{B} E\right)$. Since $A(B)$ is closed in $R_{0}$ we are done if we show that $A(E) \cap A\left(C_{B} E\right)$ is empty. But this is clear; for if $A(g)=A(e)$ for some $g \in C_{B} E$ and $e \in E$ then $g=e+k$ with $k \in K_{B}$, i.e. $g \in E$.

TheOREM (5.10). Suppose $g$ is an admissible metric, $\delta \in \mathbf{R}^{L}, a \in \mathbf{R}, s \in \mathbf{N}$, and $1<p<\infty$. There is a Banach space, $\tilde{W}_{s+2, \delta, a}^{p}\left(\wedge^{q} M, g\right)$, with the properties:

$$
\Delta_{g}: \tilde{W}_{s+2, \delta, a}^{p}\left(\bigwedge^{q} M, g\right) \rightarrow W_{s, \delta, a+2}^{p}\left(\bigwedge^{q} M, g\right)
$$

is continuous and Fredholm; $\tilde{W}_{s+2, \delta, a}^{p}\left(\bigwedge^{q} M, g\right)=W_{s+2, \delta, a}^{p}\left(\bigwedge^{q} M, g\right)$, equipped with an equivalent norm, if $\delta \in \mathbf{R}^{L}-\mathscr{D}_{\Delta}$; in general

$$
\tilde{W}_{s+2, \delta, a}^{p}\left(\bigwedge^{q} M, g\right) \subset \bigcap_{\eta<\delta} W_{s+2, \eta, a}^{p}\left(\bigwedge^{q} M, g\right) ;
$$

and the range of (5.10.1) is the closure of the range of

$$
\Delta_{g}: W_{s+2, \delta, a}^{p}\left(\wedge^{q} M, g\right) \rightarrow W_{s, \delta, a+2}^{p}\left(\wedge^{q} M, g\right) .
$$


Proof. Since $\mathscr{D}_{\Delta}$ has the form (5.2.1), almost all $\tau \leqslant \delta$ are in $\mathbf{R}^{L}-\mathscr{D}_{\Delta}$. For each such $\tau$ let $\tilde{B}_{\tau}$ be the closure of $W_{s+2, \delta, a}^{p}\left(\bigwedge^{q} M, g\right)$ in

$$
B_{\tau}=\left\{\sigma \in W_{s+2, \tau, a}^{p}\left(\bigwedge^{q} M, g\right) \mid \Delta_{g} \sigma \in W_{s, \delta, a+2}^{p}\left(\bigwedge^{q} M, g\right)\right\}
$$

with respect to the norm

$$
\|\sigma\|_{B_{\tau}}=\|\sigma\|\left(W_{s+2, \tau, a}^{p}\left(\bigwedge^{q} M, g\right)\right)+\left\|\Delta_{g} \sigma\right\|\left(W_{s, \delta, a+2}^{p}\left(\bigwedge^{q} M, g\right)\right)
$$

and equip $\tilde{B}_{\tau}$ with the norm \|\|$_{B_{\tau}}$. By the previous lemma

$$
\Delta_{g}: \tilde{B}_{\tau} \rightarrow W_{s, \delta, a+2}^{p}\left(\bigwedge^{q} M, g\right)
$$

is continuous and Fredholm with range equal to the closure of the range of (5.10.3).

We claim that all the $\tilde{B}_{\tau}$ are equal as sets and have equivalent norms. To see this first suppose $\tau_{1}<\tau_{2} \leqslant \delta$. Then there is a continuous inclusion $i: \tilde{B}_{\tau_{2}} \rightarrow \tilde{B}_{\tau_{1}}$, and so we have the commutative diagram

$$
\begin{array}{lll}
\tilde{B}_{\tau_{2}} & \stackrel{\Delta_{g}}{\rightarrow} & W_{s, \delta, a+2}^{p}\left(\bigwedge^{q} M, g\right) \\
i \downarrow & \nearrow \Delta_{g} & \\
\tilde{B}_{\tau_{1}} & &
\end{array}
$$

Because $\Delta_{g}: \tilde{B}_{\tau_{j}} \rightarrow W_{s, \delta, a+2}^{p}\left(\bigwedge^{q} M, g\right)$ is Fredholm, $i: \tilde{B}_{\tau_{2}} \rightarrow \tilde{B}_{\tau_{1}}$ also is. This means $\tilde{B}_{\tau_{2}}$ is closed in $\tilde{B}_{\tau_{1}}$. But $W_{s+2, \delta, a}^{p}\left(\bigwedge^{q} M, g\right)$ is dense in both. Hence $\tilde{B}_{\tau_{1}}$ and $\tilde{B}_{\tau_{2}}$ are equal as sets and $i$ is an isomorphism.

For general, $\tau_{1}, \tau_{2}<\delta$ and in $\mathbf{R}^{L}-\mathscr{D}_{\Delta}$ there is a $\tau_{3} \in \mathbf{R}^{L}-\mathscr{D}_{\Delta}$ such that $\tau_{1}$, $\tau_{2}<\tau_{3} \leqslant \delta$. Thus $\tilde{B}_{\tau_{2}}=\tilde{B}_{\tau_{3}}=\tilde{B}_{\tau_{1}}$ and they all have equivalent norms. Because of this we may take $\tilde{W}_{s+2, \delta, a}^{p}\left(\bigwedge^{q} M, g\right)$ to be any one of the $\tilde{B}_{\tau}$.

To see that (5.10.2) holds for $\tilde{W}_{s+2, \delta, a}^{p}\left(\wedge^{q} M, g\right)$ all we have to do is note that for $\eta<\delta$ there is a $\tau \in \mathbf{R}^{L}-\mathscr{D}_{\Delta}$ such that $\eta<\tau \leqslant \delta$. For such $\tau$ it is clear that $\tilde{B}_{\tau} \subset W_{s+2, \eta, a}^{p}\left(\bigwedge^{q} M, g\right)$.

Finally if $\delta \in \mathbf{R}^{L}-\mathscr{D}_{\Delta}$, then we have $\tilde{B}_{\delta}$. Obviously $\tilde{B}_{\delta}$ is $W_{s+2, \delta, a}^{p}\left(\bigwedge^{q} M, g\right)$ equipped with an equivalent norm.

Observe that while it is true that the range of (5.10.1) is the closure of the range of (5.10.3), the kernel of (5.10.1) may be strictly larger than that of (5.10.3). For instance if $g$ is a product metric on $\partial M_{0} \times \mathbf{R}^{+}$, then $\sigma(x) \equiv 1$ is in $\tilde{W}_{2,0}^{p}\left(\wedge^{0} M, g\right)$ though not in $W_{2,0}^{p}\left(\wedge^{0} M\right)$.

To finish this section we shall prove an analogous result for $d+d_{g}^{*}$. This result will be useful in $§ 7$. In stating it we let

$$
W_{s, \delta, a}^{p}\left(\bigwedge^{*} M, g\right)=\bigoplus_{n}^{q=0} W_{s, \delta, a}^{p}\left(\bigwedge^{q} M, g\right) \text {. }
$$

TheOREM (5.11). Suppose $g$ is admissible, $\delta \in \mathbf{R}^{L}, a \in \mathbf{R}, s \in \mathbf{N}$, and $1<p<\infty$. There is a Banach space $\tilde{\tilde{W}}_{s+1, \delta, a}^{p}\left(\Lambda^{*} M, g\right)$, with the properties:

$$
d+d_{g}^{*}: \tilde{\tilde{W}}_{s+1, \delta, a}^{p}\left(\Lambda^{*} M, g\right) \rightarrow W_{s, \delta, a+1}^{p}\left(\bigwedge^{*} M, g\right)
$$

is continuous and Fredholm; if

$$
d+d_{g}^{*}: W_{s+1, \delta, a}^{p}\left(\bigwedge^{*} M, g\right) \rightarrow W_{s, \delta, a+1}^{p}\left(\bigwedge^{*} M, g\right)
$$


is Fredholm, then $\tilde{\tilde{W}}_{s+1, \delta, a}^{p}\left(\bigwedge^{*} M, g\right)=W_{s+1, \delta, a}^{p}\left(\Lambda^{*} M, g\right)$, equipped with an equivalent norm; in general

$$
\tilde{\tilde{W}}_{s+1, \delta, a}^{p}\left(\Lambda^{*} M, g\right) \subset \bigcap_{\eta<\delta} W_{s+1, \eta, a}^{p}\left(\Lambda^{*} M, g\right) ;
$$

and the range of (5.11.1) is the closure of the range of

$$
d+d_{g}^{*}: W_{s+1, \delta, a}^{p}\left(\wedge^{*} M, g\right) \rightarrow W_{s, \delta, a+1}^{p}\left(\wedge^{*} M, g\right) .
$$

Proof. Let $\Gamma=\bigcap_{q=0}^{n} \mathscr{D}_{\Delta}(g, p, q, a-1)$ and let $\delta_{0} \in \Gamma$. Then

$$
\Delta_{g}: W_{s+2, \delta_{0}, a-1}^{p}\left(\bigwedge^{*} M, g\right) \rightarrow W_{s, \delta_{0}, a+1}^{p}\left(\bigwedge^{*} M, g\right)
$$

is Fredholm. Moreover it factors as

$$
\begin{aligned}
W_{s+2, \delta_{0}, a-1}^{p}\left(\bigwedge^{*} M, g\right) \stackrel{d+d_{g}^{*}}{\rightarrow} W_{s+1, \delta_{0}, a}^{p}\left(\bigwedge^{*} M, g\right) \\
\stackrel{d+d_{g}^{*}}{\rightarrow} W_{s, \delta_{0}, a+1}^{p}\left(\Lambda^{*} M, g\right) .
\end{aligned}
$$

Since (5.11.5) has a closed, finite codimensional range, so does

$$
d+d_{g}^{*}: W_{s+1, \delta_{0}, a}^{p}\left(\wedge^{*} M, g\right) \rightarrow W_{s, \delta_{0}, a+1}^{p}\left(\wedge^{*} M, g\right) .
$$

Because the kernel of (5.11.7) is contained in $\mathfrak{S}_{\mathfrak{E}}^{p} \delta_{0}, a$, it is also finite dimensional. Thus (5.11.7) is Fredholm for $\delta_{0} \in \Gamma$.

For any $\delta \in \mathbf{R}^{L}$ there is a $\delta_{0} \in \Gamma$ such that $\delta_{0} \leqslant \delta$. Hence we may proceed as in the previous theorem.

6. Hodge decomposition. In the last section we showed for admissible metrics that $W_{s, \delta, a}^{p}\left(\bigwedge^{q} M, g\right)=\Delta_{g} \tilde{W}_{s+2, \delta, a-2}^{p}\left(\bigwedge^{q} M, g\right) \oplus F$ with $F$ a finite dimensional space. Unlike the situation for a compact manifold, $F$ cannot always be picked to be $\mathfrak{S}_{\delta, a}^{p}\left(\wedge^{q} M, g\right)$. In fact the dimensions usually are not the same, for the dimension of $F$ is the same as that of the kernel of the adjoint of $\Delta_{g}: W_{s+2, \delta, a-2}^{p}\left(\wedge^{q} M, g\right) \rightarrow$ $W_{s, \delta, a}^{p}\left(\bigwedge^{q} M, g\right)$, and so equals $\operatorname{dim} \mathfrak{S}_{-\delta,-a}^{p^{\prime}}\left(\bigwedge^{q} M, g\right)$ where $1 / p+1 / p^{\prime}=1$. Since any $\sigma \in \mathfrak{S}_{-\delta,-a}^{p^{\prime}}\left(\bigwedge^{q} M, g\right)$ that is also in $W_{s, \delta, a}^{p}\left(\bigwedge^{q} M, g\right)$ may be put in $F$, this suggests that $F$ can be chosen to be $\mathfrak{S}_{-\delta,-a}^{p^{\prime}}\left(\bigwedge^{q} M, g\right)$. However, that can only be done if $\mathfrak{S}_{-\delta,-a}^{p^{\prime}}\left(\bigwedge^{q} M, g\right) \subset W_{s, \delta, a}^{p}\left(\bigwedge^{q} M, g\right)$. In the next theorem sufficient conditions are provided for this to be true.

Theorem (6.1). For $1<p<\infty, \delta \in \mathbf{R}^{L}, \quad a \in \mathbf{R}, \quad s \in \mathbf{N}$, and $g$ admissible $\mathfrak{S}_{-}^{p^{\prime},-a}\left(\bigwedge^{q} M, g\right) \subset W_{s, \delta, a}^{p}\left(\bigwedge^{q} M, g\right)$ if any of the following is true:

$$
p \geqslant 2 \text { and } \delta z+\left(a+n / 2-n / p^{\prime}\right) \rho \text { is bounded above, }
$$
$p<2$ and $(\delta+\varepsilon) z+\left(a+n / 2-n / p^{\prime}\right) \rho$ is bounded above for some $\varepsilon>0$,

Proof. All we need to do is show that $\mathfrak{S}_{-\delta,-a}^{p^{\prime}}\left(\bigwedge^{q} M, g\right) \subset W_{0, \delta, a}^{p}\left(\bigwedge^{q} M, g\right)$, for then it is in $\mathscr{S}_{\delta, a}^{p}\left(\bigwedge^{q} M, g\right)$, which by (5.3) is in $W_{s, \delta, a}^{p}\left(\bigwedge^{q} M, g\right)$ for all $s$. In the case of (6.13) this is a result of the fact that $W_{0,-\delta, 0}^{p^{\prime}}\left(\bigwedge^{q} M, g\right) \subset W_{0, \delta, 0}^{p}\left(\bigwedge^{q} M, g\right)$, which follows easily from Hölder's inequality. 
As for the first two cases, note that according to Theorem (4.7) we can, for large enough $t$, continuously imbed $W_{t,-\delta,-a}^{p^{\prime}}\left(\bigwedge^{q} M, g\right)$ into $W_{0, \hat{\delta},-a+n / p^{\prime}-n / p}^{p}\left(\bigwedge^{q} M, g\right)$ provided either

(i) $p \geqslant 2$ and $\hat{\delta} \leqslant-\delta$ or

(ii) $p<2$ and $\hat{\delta}<-\delta$.

Hence $\widetilde{S}_{-\delta,-a}^{p^{\prime}}\left(\bigwedge^{q} M, g\right) \subset W_{0, \hat{\delta},-a+n / p^{\prime}-n / p}^{p}\left(\bigwedge^{q} M, g\right)$ when either of these conditions hold.

Since

$$
\delta z+a \rho=(\delta-\hat{\delta}) z+2\left(a+n / 2-n / p^{\prime}\right) \rho+\hat{\delta} z+\left(-a+n / p^{\prime}-n / p\right) \rho,
$$

Hölder's inequality implies that $W_{0, \hat{\delta},-a+n / p^{\prime}-n / p}\left(\bigwedge^{q} M, g\right) \subset W_{0, \delta, a}^{p}\left(\bigwedge^{q} M, g\right)$ if $(\delta-\hat{\delta}) z+2\left(a+n / 2-n / p^{\prime}\right) \rho$ is bounded above. For $p \geqslant 2$ we may pick $\hat{\delta}=-\delta$, but for $p<2$ we must pick $\hat{\delta}=-\delta-\varepsilon$ for some $\varepsilon>0$. Hence we have conditions (6.1.1) and (6.1.2).

Corollary (6.2). Given $a \in \mathbf{R}, 1<p<\infty$, and an admissible metric $g$ there is a $\delta_{0} \in \mathbf{R}^{L}$ with the property that

(6.2.1) $\quad W_{s, \delta, a}^{p}\left(\bigwedge^{q} M, g\right)=\Delta_{g}\left(\tilde{W}_{s+2, \delta, a-2}^{p}\left(\bigwedge^{q} M, g\right)\right) \oplus \mathscr{S}_{-\delta,-a}^{p^{\prime}}\left(\bigwedge^{q} M, g\right)$

for all $\delta<\delta_{0}$.

Proof. A consequence of Theorem (2.9) is that $|\rho(z)| \leqslant c z$ on $M_{\infty}$ for some $c$. Thus (6.11) and (6.12) can be satisfied by picking $\delta$ sufficiently negative on each end.

Corollary (6.3). For admissible $g$ the decomposition

$$
L^{p}\left(\bigwedge^{q} M, g\right)=\Delta_{g}\left(\tilde{W}_{2,0-2}^{p}\left(\bigwedge^{q} M, g\right)\right) \oplus \mathfrak{S}_{0,0}^{p^{\prime}}\left(\bigwedge^{q} M, g\right)
$$

holds if any of the following are true:

$$
\begin{gathered}
p=2 \\
p \geqslant 2 \text { and } \rho \text { is bounded below, } \\
p<2 \text { and } \int_{M} d V_{g}<\infty
\end{gathered}
$$

If $g$ is admissible but not complete, then $\mathfrak{S}_{\mathcal{E}}^{p^{\prime}, 0}\left(\bigwedge^{q} M, g\right)$ can have elements that are not closed or coclosed. However, $\delta$ can be picked so that every element of $\mathfrak{S}_{-\delta,-a}^{p^{\prime}}\left(\bigwedge^{q} M, g\right)$ is closed and coclosed.

Proposition (6.4). For $a \in \mathbf{R}$ and $g$ admissible, there is $a \hat{\delta} \in \mathbf{R}^{L}$ such that every element of $\mathfrak{S}_{-\delta,-a}^{p^{\prime}}\left(\bigwedge^{q} M, g\right)$ is closed and coclosed if $\delta<\hat{\delta}$.

Proof. As a result of Theorem (2.9) there is a $\hat{\delta} \in \mathbf{R}^{L}$ such that

$$
\hat{\delta} z+\left((a-1)+n / 2-n / p^{\prime}\right) \rho
$$

is bounded above. Thus by the proof of (6.1) we have, for large enough $t$, a continuous inclusion

$$
W_{t,-\delta,-a+1}^{p^{\prime}}\left(\bigwedge^{r} M, g\right) \subset W_{0, \delta, a-1}^{p}\left(\bigwedge^{r} M, g\right)
$$

for all $r$ and all $\delta<\hat{\delta}$. 
Let $\sigma \in \mathfrak{S}_{-\delta,-a}^{p^{\prime}}\left(\bigwedge^{q} M, g\right)$. Since $C_{0}^{\infty}\left(\bigwedge^{q} M\right)$ is dense in $W_{t+1,-\delta,-a}^{p^{\prime}}\left(\bigwedge^{q} M, g\right)$, there is a sequence $\left\{\phi_{i}\right\} \subset C_{0}^{\infty}\left(\wedge^{q} M\right)$ that converges to $\sigma$ in $W_{t+1,-\delta,-a}^{p^{\prime}}\left(\wedge^{q} M, g\right)$. From the continuity of $d$ and $d_{g}^{*}$, we get that $d \phi_{i} \rightarrow d \sigma$ in $W_{t,-\delta,-a+1}^{p^{\prime}}\left(\wedge^{q+1} M, g\right)$ and $d_{g}^{*} \phi_{i} \rightarrow d_{g}^{*} \sigma$ in $W_{t,-\delta,-a+1}^{p^{\prime}}\left(\bigwedge^{q-1} M, g\right)$. Hence by (6.4.1) $d \phi_{i} \rightarrow d \sigma$ in $W_{0, \delta, a-1}^{p}\left(\bigwedge^{q} M, g\right)$ and $d_{g}^{*} \phi_{i} \rightarrow d_{g}^{*} \sigma$ in $W_{0, \delta, a-1}^{p}\left(\wedge^{q-1} M, g\right)$. It follows from this and the duality of the spaces $W_{0,-\delta,-a+1}^{p^{\prime}}\left(\bigwedge^{r} M, g\right)$ and $W_{0, \delta, a-1}^{p}\left(\bigwedge^{r} M, g\right)$ that

$$
\int_{M}\langle d \sigma, d \sigma\rangle_{g} d V_{g} \text { and } \int_{M}\left\langle d_{g}^{*} \sigma, d_{g}^{*} \sigma\right\rangle_{g} d V_{g}
$$

are finite and that

$$
\int_{M}\langle d \sigma, d \sigma\rangle_{g} d V_{g}=\lim _{i \rightarrow \infty} \int_{M}\left\langle d \sigma, d \phi_{i}\right\rangle_{g} d V_{g}
$$

and

$$
\int_{M}\left\langle d_{g}^{*} \sigma, d_{g}^{*} \sigma\right\rangle_{g} d V_{g}=\lim _{i \rightarrow \infty} \int_{M}\left\langle d_{g}^{*} \sigma, d_{g}^{*} \phi_{i}\right\rangle_{g} d V_{g} .
$$

But Stokes's theorem implies that the integrals on the right-hand side of (6.4.2) and (6.4.3) are zero for $\sigma \in \breve{S}_{-\delta,-a}^{p^{\prime}}\left(\bigwedge^{q} M, g\right)$.

In the next theorem we deal with a larger class of metrics than the admissible ones; namely those equivalent to admissible ones in the following sense.

Definition (6.5). Two metrics, $g_{0}$ and $g_{1}$, are equivalent if there is a constant $c>0$ such that $g_{0} / c \leqslant g_{1} \leqslant c g_{0}$. This relation is denoted $g_{0} \approx g_{1}$.

Notice that if $g_{0} \approx g_{1}$, then $L^{p}\left(\wedge^{q} M, f d V_{g_{0}}\right)$ and $L^{p}\left(\wedge^{q} M, f d V_{g_{1}}\right)$ are equal as vector spaces and have equivalent norms for any continuous $f: M \rightarrow \mathbf{R}^{+}$. Also note that equivalent metrics can have quite different curvature properties. For instance on $S^{1} \times \mathbf{R}$ the metrics $d \theta^{2}+d x^{2}$ and $\left(2+\sin \left(e^{x^{2}}\right)\right)\left(d \theta^{2}+d x^{2}\right)$ are equivalent.

THEOREM (6.6). In addition to the conditions already assumed for $M$, suppose $M$ is oriented. Let $g_{1}$ be a metric on $M$ that is equivalent to an admissible metric $g$. There is $a \bar{\delta} \in \mathbf{R}^{L}$ such that if $\delta<\bar{\delta}$ and $\phi \in W_{0, \delta, 0}^{p}\left(\bigwedge^{0} M, g\right)$, then $\phi=d_{g_{1}}^{*} \sigma$ for some $\sigma \in \bigcap_{\tau<\delta} W_{0, \tau,-1}^{p}\left(\bigwedge^{1} M, g\right)$.

Proof. By (6.2) and (6.4) there is a $\hat{\delta}$ such that if $\delta<\hat{\delta}$ then

$$
W_{0, \delta, 0}^{p}\left(\wedge^{0} M, g\right)=d_{g}^{*} d\left(\tilde{W}_{2, \delta,-2}^{p}\left(\wedge^{0} M, g\right)\right) \oplus \mathfrak{S}_{-\delta, 0}^{p^{\prime}}\left(\wedge^{0} M, g\right)
$$

with $\mathfrak{S}_{-}^{p^{\prime}} \underset{\delta, 0}{ }$ containing only constants. Picking $\bar{\delta} \leqslant \hat{\delta}$ so that $\int_{M} e^{-p \bar{\delta} z} d V_{g}=\infty$, we get

$$
W_{0, \delta, 0}^{p}\left(\bigwedge^{0} M, g\right)=d_{g}^{*} d\left(\tilde{W}_{2, \delta,-2}^{p}\left(\bigwedge^{0} M, g\right)\right)
$$

for $\delta<\bar{\delta}$.

Since $g_{1}$ is equivalent to $g$, the isomorphism

$$
{ }_{g_{1}}^{*}{ }_{g}: \wedge^{q} M \rightarrow \wedge^{q} M
$$

extends to an isomorphism

$$
{ }_{g_{1}}^{*}{ }_{g}^{*}: W_{0, \tau, a}^{p}\left(\bigwedge^{q} M, g\right) \rightarrow W_{0, \tau, a}^{p}\left(\bigwedge^{q} M, g\right) .
$$


Hence the solution to $\phi=d_{g_{1}}^{*}$ we seek is $\sigma=*_{g_{1}} *_{g} \tilde{\sigma}$ with $\tilde{\sigma}$ the solution to $(-1)^{n-1} *_{g} *_{g_{1}} \phi=d_{g}^{*} \tilde{\sigma}$. Since $\tilde{\sigma}$ can be picked to be $d F$ for some $F \in \tilde{W}_{2, \delta,-2}^{p}\left(\bigwedge^{0} M, g\right)$, it follows from Theorem (5.10) that $\tilde{\sigma}$ has all the properties we want. Hence by (6.6.4) $\sigma$ also does.

7. Hodge representation. We now turn our attention to determining the dimension of the space of closed and coclosed forms in $L^{2}$ and determining to what extent its elements represent de Rham cohomology. The key result is Theorem (7.4), which holds for many admissible and nonadmissible metrics. In the theorem we do not assume $g$ is admissible.

Notation (7.1). Let $g_{0}$ be a metric on $M$ that is a product metric on $M_{\infty}$. If $g$ is a metric on $M$, then $F_{g}=\left(\operatorname{det} g / \operatorname{det} g_{0}\right)^{1 / 2}$. Thus $d V_{g}=F_{g} d V_{g_{0}}$.

Notation (7.2). For $0 \leqslant t$ the function $\mathscr{M}_{t}: \partial M_{0} \times \overline{\mathbf{R}}^{+} \rightarrow \partial M_{0} \times \overline{\mathbf{R}}^{+}$is given by $(\omega, z) \rightarrow(\omega, t z)$.

Definition (7.3). A metric $g$ on $M$ is $q$-bounded above if it is conformal to an asymptotically translation invariant one and if for every $0 \leqslant t \leqslant 1$, every $(\omega, z) \in$ $M_{\infty}$, and every $\nu \in \wedge^{q} M_{(\omega, z t)}$ the inequality

$$
\left\|d z \wedge\left(\mathscr{M}_{t}^{*} \nu\right)(\omega, z)\right\|_{g(\omega, z)}^{2} F_{g}(\omega, z) \leqslant c\|(d z \wedge \nu)(\omega, t z)\|_{g(\omega, t z)}^{2} F_{g}(\omega, t z)
$$

holds for some $c$ independent of $\nu, t$, and $(\omega, z)$.

THEOREM (7.4). Suppose $g$ is q-bounded above. If $\sigma \in L^{2}\left(\wedge^{q} M, g\right) \cap d C^{\infty}\left(\wedge^{q-1} M\right)$, then $\sigma \in \overline{d C_{0}^{\infty}\left(\wedge^{q-1} M\right)}$, the closure of $d C_{0}^{\infty}\left(\wedge^{q-1} M\right)$ in $L^{2}\left(\wedge^{q} M, g\right)$.

Proof. Let $\sigma=d \psi$ for some $\psi \in C^{\infty}\left(\bigwedge^{q-1} M\right)$. With $\phi_{R}$ as in Lemma (3.11), we have $\sigma=d \phi_{1} \psi+d\left(1-\phi_{1}\right) \psi$. Since $d \phi_{1} \psi \in d C_{0}^{\infty}\left(\wedge^{q-1} M\right)$, all we need to do is show that $\sigma_{\infty}=d\left(1-\phi_{1}\right) \psi$ is in $\overline{d C_{0}^{\infty}\left(\Lambda^{q-1} M\right)}$. Now $\left(1-\phi_{1}\right)$ has its support in $\partial M_{0} \times[1, \infty)$ and there $\sigma_{\infty}=\eta+\nu \wedge d z$ with $\eta(\omega, z) \in C^{\infty}\left(\wedge^{q} \partial M_{0}\right)$ and $\nu \in$ $C^{\infty}\left(\wedge^{q-1} \partial M_{0}\right)$. Define $\mu(\omega, z)$ by

$$
\mu(\omega, z)= \begin{cases}(-1)^{q-1} \int_{0}^{1} z\left(\mathscr{M}_{t}^{*} \nu\right)(\omega, z) d t & \text { on } M_{\infty} \\ 0 & \text { on } M_{0}\end{cases}
$$

Then $\mu \in C^{\infty}\left(\wedge^{q-1} M\right)$, and, as an easy calculation shows, $d \mu=\sigma_{\infty}$.

Setting $\mu_{R}=\phi_{R} \mu$, we get

$$
d \mu_{R}=\frac{(-1)^{q+1}}{R} \tilde{\phi}^{\prime}\left(\frac{z}{R}\right) \int_{0}^{1} z d z \wedge\left(\mathscr{M}_{t}^{*} \nu\right)(\omega, z) d t+\phi_{R} \sigma_{\infty}
$$

with $\tilde{\phi}$ as in (3.11). Clearly $\phi_{R} \sigma_{\infty} \rightarrow \sigma_{\infty}$ in $L^{2}\left(\bigwedge^{q} M, g\right)$ as $R \rightarrow \infty$. Therefore, to finish the proof we must show the convergence to 0 in $L^{2}\left(\wedge^{q} M, g\right)$ as $R \rightarrow \infty$ of

$$
\frac{1}{R} \tilde{\phi}^{\prime}\left(\frac{z}{R}\right) \int_{0}^{1} z d z \wedge\left(\mathscr{M}_{t}^{*} \nu\right)(\omega, z) d t=\lambda_{R}(\omega, z)
$$


Because of the fact that $\operatorname{supp} \tilde{\phi}^{\prime}(z / R) \subset \partial M_{0} \times[R, 2 R] \equiv M_{R}$,

$$
\begin{aligned}
\left\|\lambda_{R}\right\|_{2} & =\left(\int_{M_{R}} \frac{z^{2}}{R^{2}}\left(\tilde{\phi}^{\prime}\left(\frac{z}{R}\right)\right)^{2}\left\|\int_{0}^{1} d z \wedge\left(\mathscr{M}_{t}^{*} \nu\right)(\omega, z) d t\right\|_{g(\omega, z)}^{2} d V_{g}\right)^{1 / 2} \\
& \leqslant c\left(\int_{M_{R}}\left\|\int_{0}^{1} d z \wedge\left(\mathscr{M}_{t}^{*} \nu\right)(\omega, z) d t\right\|_{g(\omega, z)}^{2} d V_{g}\right)^{1 / 2} .
\end{aligned}
$$

In turn, Minkowski's inequality applied to the last integral in (7.4.4) gives

$$
\left\|\lambda_{R}\right\|_{2} \leqslant c \int_{0}^{1}\left(\int_{\partial M_{0}} \int_{R}^{2 R}\left\|d z \wedge\left(\mathscr{M}_{t}^{*} \nu\right)(\omega, z)\right\|_{g(\omega, z)}^{2} F_{g}(\omega, z) d z d \omega\right)^{1 / 2} d t
$$

Since $g$ is $q$-bounded above, it follows from (7.4.5) that

$$
\left\|\lambda_{R}\right\|_{2} \leqslant c \int_{0}^{1}\left(\int_{\partial M_{0}} \int_{R}^{2 R}\|d z \wedge \nu\|_{g(\omega, t z)}^{2} F_{g}(\omega, t z) d z d \omega\right)^{1 / 2} d t .
$$

The change of variable, $\xi=z t$, in (7.4.6) then yields

$$
\begin{aligned}
\left\|\lambda_{R}\right\|_{2} \leqslant & c \int_{0}^{1} t^{-1 / 2}\left(\int_{\partial M_{0}} \int_{t R}^{2 t R}\|d z \wedge \nu\|_{g}^{2} F_{g} d \xi d \omega\right)^{1 / 2} d t \\
\leqslant & c \int_{0}^{R^{-1 / 2}} t^{-1 / 2}\|\nu \wedge d z\|_{2} d t \\
& +c \int_{R^{-1 / 2}}^{1} t^{-1 / 2}\left(\int_{\partial M_{0} \times[\sqrt{R}, 2 R]}\|\nu \wedge d z\|_{g}^{2} d V_{g}\right)^{1 / 2} d t \\
= & c R^{-1 / 4}\|\nu \wedge d z\|_{2}+c\left(\int_{\partial M_{0} \times[\sqrt{R}, 2 R]}\|\nu \wedge d z\|_{g}^{2} d V_{g}\right)^{1 / 2} .
\end{aligned}
$$

This last quantity obviously goes to 0 as $R \rightarrow \infty$.

COROLlary (7.5). Let

$$
\tilde{\mathfrak{S}}^{2}\left(\bigwedge^{q} M, g\right)=\left\{\sigma \in L^{2}\left(\bigwedge^{q} M, g\right) \mid d \sigma=d_{g}^{*} \sigma=0\right\} .
$$

If $g$ is q-bounded above, then

$$
\operatorname{dim} \tilde{\mathfrak{S}}^{2}\left(\bigwedge^{q} M, g\right) \leqslant \operatorname{dim} H_{D R}^{q}(M) .
$$

Proof. By the theorem of Kodaira mentioned in the introduction, the space of closed forms in $L^{2}\left(\bigwedge^{q} M, g\right)$ is the orthogonal direct sum

$$
\overline{d C_{0}^{\infty}\left(\bigwedge^{q-1} M\right)} \oplus \tilde{\mathscr{S}}^{2}\left(\bigwedge^{q} M, g\right) .
$$

Thus by Theorem (7.4) there are no exact forms in $\tilde{\mathscr{S}}^{2}\left(\wedge^{q} M, g\right)$.

Examples of $q$-bounded above metrics were given in the introduction. In the case that $g$ is equivalent to a $q$-bounded above metric that is also admissible, we may use the Fredholm results of the previous sections to obtain a precise determination of $\tilde{\mathfrak{S}}^{2}\left(\bigwedge^{q} M, g\right)$. 
THEOREM (7.6). Suppose that $g_{1}$ is both q-bounded above and admissible and that $g$ is equivalent to $g_{1}$. Every $v \in H_{D R}^{q}(M)$ with a representative in $L^{2}\left(\wedge^{q} M, g\right)$ has a unique representative in $\tilde{\mathfrak{S}}^{2}\left(\bigwedge^{q} M, g\right)$.

Proof. Once again the equivalence of $g$ and $g_{1}$ means $L^{2}\left(\wedge^{q} M, g\right)=L^{2}\left(\bigwedge^{q} M, g_{1}\right)$ with equivalent norms. Taking $\tilde{W}_{1,0,-1}^{2}\left(\Lambda^{*} M, g_{1}\right)$ to be as in Theorem (5.11) and $\tilde{\tilde{W}}_{1,0,-1}^{2}\left(\bigwedge^{q-1} M, g_{1}\right)$ to be the $q-1$ forms in $\tilde{W}_{1,0,-1}^{2}\left(\bigwedge^{*} M, g_{1}\right)$, we have from that theorem that $d \tilde{\tilde{W}}_{1,0,-1}^{2}\left(\bigwedge^{q-1} M, g_{1}\right)$ is closed in $L^{2}\left(\bigwedge^{q} M, g\right)$. Since $C_{0}^{\infty}\left(\bigwedge^{q-1} M\right) \subset$ $\tilde{\tilde{W}}_{1,0,-1}^{2}\left(\bigwedge^{q-1} M, g_{1}\right)$ this means that

$$
\overline{d C_{0}^{\infty}\left(\bigwedge^{q-1} M\right)}=d \tilde{\tilde{W}}_{1,0,-1}^{2}\left(\bigwedge^{q-1} M, g_{1}\right) .
$$

In other words every element of $\overline{d C_{0}^{\infty}\left(\wedge^{q-1} M\right)}$ is exact.

The theorem then follows from Kodaira's theorem and the fact that no element of $\tilde{\mathfrak{S}}^{2}\left(\wedge^{q} M, g_{1}\right)$ is exact.

Naturally, the question arises as to which cohomology classes have representatives in $L^{2}\left(\wedge^{q} M, g\right)$. We answer this in the next theorem. But first some terminology needs to be established.

Definition (7.7). For $\Omega_{l}$ a component of $\partial M_{0}$, a cohomology class $v \in H_{D R}^{q}(M)$ is said to have compact support on $\Omega_{l} \times \mathbf{R}^{+}$if there is a representative, $\sigma$, of $v$ such that $\sigma \equiv 0$ on $\Omega_{l} \times[R, \infty)$ for some $R \geqslant 0$.

Definition (7.8). Let $\Omega_{1}, \ldots, \Omega_{L}$ be the components of $\partial M_{0}$. Also take $C^{\infty}\left(\wedge^{q} M\right)_{\mathbf{R}^{+}}$to be the set of $\phi \in C^{\infty}\left(\bigwedge^{q} M\right)$ that are $\mathbf{R}^{+}$invariant on $M_{\infty}$. For a metric $g$

$$
\mathscr{A}(g ; q)=\left\{l \mid \int_{\Omega_{l} \times \mathbf{R}^{+}}\|\phi\|_{g}^{2} d V_{g}=\infty \text { for some } \phi \in C^{\infty}\left(\bigwedge^{q} M\right)_{\mathbf{R}^{+}}\right\}
$$

Observe that in the case $g=e^{2 \rho} h$ is admissible

$$
\mathscr{A}(g, q)=\left\{l \mid e^{-2 q \rho} \notin L^{1}\left(\Omega_{l} \times \mathbf{R}^{+}, d V_{g}\right)\right\} .
$$

THEOREM (7.9). Suppose $g_{1}=e^{2 \rho} h$ is admissible and $g \approx g_{1}$. With $\mathscr{A}$ and $\Omega_{l}$ as in (7.8), set $M_{\infty}^{\infty}(g ; q)=\bigcup_{l \in \mathscr{A}} \Omega_{l} \times \mathbf{R}^{+}$. The cohomology classes in $H_{D R}^{q}(M)$ with representatives in $\tilde{\mathfrak{S}}^{2}\left(\bigwedge^{q} M, g\right)$ are exactly those that have compact support on $M_{\infty}^{\infty}(g ; q)$.

Proof. As in Theorem (7.6), the equivalence of $g$ and $g_{1}$, combined with Kodaira's theorem and Theorem (5.11) implies that the classes in $H_{D R}^{q}(M)$ with representatives in $\tilde{\mathscr{S}}^{2}\left(\bigwedge^{q} M, g\right)$ are the same as those with representatives in $\tilde{\mathfrak{S}}^{2}\left(\wedge^{q} M, g_{1}\right)$. Hence we only need to prove the theorem for $g_{1}$.

According to Theorem (2.9) there is a $\bar{\rho} \in C^{\infty}(M)$ that is a function only of $z$ on $M_{\infty}$ and an asymptotically translation invariant metric $\bar{h}$ such that $g=e^{2 \bar{\rho}} \bar{h}$. By Propositions (2.5) and (2.6) $\bar{\rho}$ satisfies condition (2.3.1) for some $\theta$ and any asymptotically translation invariant metric. In particular if $h_{0}$ is any metric that on $M_{\infty}$ is the product metric $d s_{\partial M_{0}}^{2}+d z^{2}$, then $e^{2 \bar{\rho}} h_{0}$ is admissible. Since $g_{1}$ is equivalent to $e^{2 \bar{\rho}} h_{0}$, we can use the argument in the preceding paragraph to see that we only have to prove the theorem for metrics of the form $e^{2 \bar{\rho}} h_{0}$. 
Thus without loss of generality suppose $g$ is an admissible metric and that $g=e^{2 \rho} h_{0}$ with $\rho$ a function only of $z$ on $M_{\infty}$ and $h_{0}=d s_{\partial M_{0}}^{2}+d z^{2}$ on $M_{\infty}$. Let $\tilde{\Delta}$ be the Laplacian on $\partial M_{0}$ associated with $d s_{\partial M_{0}}^{2}, \tilde{d}$ the exterior derivative, and $\tilde{d}^{*}$ the coderivative. Take $\left\{\eta_{-1 i}\right\} \cup\left\{\eta_{0 i}\right\} \cup\left\{\eta_{1 i}\right\} \cup \cdots \cup\left\{\eta_{L i}\right\}$ to be an orthonormal basis of $L^{2}\left(\wedge^{q-1} \partial M_{0}, d s_{\partial M_{0}}^{2}\right)$ consisting of eigenforms of $\tilde{\Delta}$ with the $\eta_{-1 i}$ closed but not coclosed, the $\eta_{0 i}$ coclosed but not closed, and the $\eta_{l i}$ harmonic and equal to 0 on $\Omega_{j}$ for $j \neq l$. Say $\tilde{\Delta} \eta_{01}=\lambda_{0 i}^{2} \eta_{0 i}$. If $\left\{\psi_{-1 i}\right\}$ is an orthonormal basis for the coclosed nonharmonic forms in $L^{2}\left(\bigwedge^{q} \partial M_{0}, d s_{\partial M_{0}}^{2}\right)$ and $\left\{\psi_{l i}\right\}$ is an orthonormal basis for the harmonic $q$-forms on $\partial M_{0}$ such that $\psi_{l i} \equiv 0$ on $\Omega_{j}$ for $j \neq l$, then $\left\{\psi_{-1 l}\right\} \cup$ $\left\{\lambda_{0 i}^{-1} \tilde{d} \eta_{0 i}\right\} \cup\left\{\psi_{1 i}\right\} \cup \cdots \cup\left\{\psi_{L i}\right\}$ is an orthonormal basis for $L^{2}\left(\bigwedge^{q} \partial M_{0}, d s_{\partial M_{0}}^{2}\right)$.

Every $\sigma \in L^{2}\left(\bigwedge^{q} M_{\infty}, g\right)$ has an orthogonal series expansion of the form

$$
\begin{aligned}
\sigma= & \sum_{l=-1}^{L} \sum_{i} f_{l i}(z) d z \wedge \eta_{l i}+\sum_{i} h_{-1 i}(z) \psi_{-1 i} \\
& +\sum_{i} h_{0 i}(z) \lambda_{0 i}^{-1} \tilde{d} \eta_{0 i}+\sum_{l=1}^{L} \sum_{i} h_{l i}(z) \psi_{l i} .
\end{aligned}
$$

From this we see that

$$
\begin{aligned}
d \sigma= & \sum_{i}\left(h_{0 i}^{\prime}(z) \lambda_{0 i}^{-1}-f_{0 i}(z)\right) d z \wedge \tilde{d} \eta_{0 i}+\sum_{i} h_{-1 i}^{\prime}(z) d z \wedge \psi_{-1 i} \\
& +\sum_{i} h_{-1 i}(z) \tilde{d} \psi_{-1 i}+\sum_{l=1}^{L} \sum_{i} h_{l i}^{\prime}(z) d z \wedge \psi_{l i} .
\end{aligned}
$$

If $d \sigma=0$, then it follows that

$$
h_{-1 i} \equiv 0, h_{0 i}^{\prime}=\lambda_{0 i} f_{0 i}, \text { and } h_{l i}=a_{l i} \in \mathbf{R} \text { for } l>0 .
$$

Similarly we have

$$
\begin{aligned}
d^{*} \sigma= & -\sum_{i}\left[f_{-1 i}(z) e^{(n-2 q) \rho}\right]^{\prime} e^{(2 q-n) \rho} \eta_{-1 i}-\sum_{i} f_{-1 i}(z) d z \wedge \tilde{d}^{*} \eta_{-1 i} \\
& +\sum_{i}\left(h_{0 i}(z) \lambda_{0 i} e^{(n-2 q) \rho}-\left[f_{0 i}(z) e^{(n-2 q) \rho}\right]^{\prime}\right) e^{(2 q-n) \rho} \eta_{0 i} \\
& -\sum_{l=1}^{L} \sum_{i}\left[f_{l i}(z) e^{(n-2 q) \rho}\right]^{\prime} e^{(2 q-n) \rho} \eta_{l i} .
\end{aligned}
$$

If $d^{*} \sigma=0$, then it follows that

$$
\begin{aligned}
& f_{-1 i} \equiv 0, \quad\left[f_{0 i}(z) e^{(n-2 q) \rho}\right]^{\prime}= \lambda_{0 i} h_{0 i}(z) e^{(n-2 q) \rho}, \\
& \text { and } f_{l i}=b_{l i} e^{(2 q-n) \rho} \quad \text { for } l>0 \text { and } b_{l i} \in \mathbf{R} .
\end{aligned}
$$

As a result of (7.9.3) and (7.9.5) we have that if $\sigma \in \tilde{\mathfrak{S}}^{2}\left(\bigwedge^{q} M, g\right)$, then on $M_{\infty}$

$$
\sigma=d\left[\sum_{i} h_{0 i}(z) \lambda_{0 i}^{-1} \eta_{01}+\sum_{l=1}^{L} \sum_{i} b_{l i} \int_{0}^{z} e^{(2 q-n) \rho(\zeta)} d \zeta \eta_{l i}\right]+\sum_{l=1}^{L} \sum_{i} a_{l i} \psi_{l i}
$$

with $h_{0 i}$ satisfying $h_{0 i}^{\prime \prime}+(n-2 q) \rho^{\prime} h_{01}^{\prime}-\lambda_{0 i}^{2} h_{0 i}=0$. 
In order for $\sigma$ to be in $L^{2}\left(\wedge^{q} M, g\right)$ each term in (7.9.1) must be in $L^{2}\left(\wedge^{q} M_{\infty}, g\right)$. Therefore the $a_{l i}$ in (7.9.6) must be 0 if $l \in \mathscr{A}(g ; q)$ and may be anything if $l \notin \mathscr{A}(g ; q)$. Hence letting

$$
\tilde{\beta}=\sum_{i} h_{0 i}(z) \lambda_{0 i}^{-1} \eta_{0 i}+\sum_{l=1}^{L} \sum_{i} b_{l i} \int_{0}^{z} e^{(2 q-n) \rho(\zeta)} d \zeta \eta_{l i}
$$

on $\partial M_{0} \times[1, \infty)$ and letting $\beta$ be a $C^{\infty}$ extension of $\tilde{\beta}$ to all of $M$ we get that on $\partial M_{0} \times[1, \infty)$

$$
\sigma-d \beta=\sum_{l \notin \mathscr{A}} \sum_{i} a_{l i} \psi_{l i} .
$$

This means that the only classes in $H_{D R}^{q}(M)$ with representatives in $\tilde{\mathscr{S}}^{2}\left(\bigwedge^{q} M, g\right)$ are the ones that are compactly supported on $M_{\infty}^{\infty}(g ; q)$. On the other hand if $\nu \in H_{D R}^{q}(M)$ is such a class and $\phi$ is a representative of $\nu$, then by de Rham's theorem on $M_{\infty}$

$$
\phi=\sum_{l \notin \mathscr{A}} \sum_{i} a_{l i} \psi_{l i}+d \gamma_{0} .
$$

Choosing $\gamma$ to be a $C^{\infty}$ extension to all of $M$ of the restriction of $\gamma_{0}$ to $\partial M_{0} \times\{1, \infty\}$, we see that $\nu$ has a representative that is $\sum_{l \notin \mathscr{A}} \sum_{i} a_{l i} \psi_{l i}$ on $M_{\infty}$, namely $\phi-d \gamma$. Thus $\nu$ has a representative in $L^{2}\left(\wedge^{q} M, g\right)$. It follows, therefore, from Kodaira's theorem and Theorem (5.11) that $\nu$ has a representative in $\tilde{\mathscr{S}}^{2}\left(\bigwedge^{q} M, g\right)$.

COROllaRY (7.10). If $g \approx g_{1}$ and $g_{1}$ is admissible and $q$-bounded above, then the classes of $H_{D R}^{q}(M)$ with representatives in $\tilde{\mathfrak{S}}^{2}\left(\wedge^{q} M, g\right)$ are exactly those compactly supported on $M_{\infty}^{\infty}(g ; q)$. Moreover the representation is unique.

8. $L^{2}$-cohomology. In this section we give a few applications of the results of the preceding sections to $L^{2}$-cohomology. Recently this cohomology has received quite a bit of attention; in part, because of its use in the study of singular algebraic varieties (see [4, 5 and 13]). We begin by defining various subspaces of $L^{2}$.

DEFINITION 8.1. For an arbitrary Riemannian manifold $X$

$$
\begin{gathered}
L_{e}^{2}\left(\wedge^{q} X\right)=\text { the closure of } d C_{0}^{\infty}\left(\wedge^{q-1} X\right) \text { in } L^{2}\left(\wedge^{q} X\right), \\
L_{c e}^{2}\left(\wedge^{q} X\right)=\text { the closure of } d_{g}^{*} C_{0}^{\infty}\left(\wedge^{q+1} X\right) \text { in } L^{2}\left(\wedge^{q} X\right), \\
\mathfrak{Q}^{q}(X)=\left\{\sigma \in L^{2}\left(\bigwedge^{q} X\right) \mid d \sigma \in L^{2}\left(\wedge^{q+1} X\right)\right\}, \\
\mathfrak{Q}_{c e}^{q}(X)=\mathfrak{\Omega}^{q}(X) \cap L_{c e}^{2}\left(\wedge^{q} X\right) .
\end{gathered}
$$

We equip $L_{e}^{2}\left(\bigwedge^{q} X\right)$ and $L_{c e}^{2}\left(\bigwedge^{q} X\right)$ with the $L^{2}$ norm and equip $\mathfrak{R}^{q}(X)$ and $\mathfrak{R}_{c e}^{q}(X)$ with the norm $\|\sigma\|_{\mathfrak{R}}=\left(\|\sigma\|_{2}^{2}+\|d \sigma\|_{2}^{2}\right)^{1 / 2}$. So equipped each space is a sub-Hilbert space of $L^{2}$.

DEFINITION (8.2). The $q$ th- $L^{2}$-cohomology group of $X$ is

$$
H_{2}^{q}(X)=\left[\operatorname{Ker} d: \mathfrak{\Omega}^{q}(X) \rightarrow \mathfrak{2}^{q+1}(X)\right] / d \mathfrak{\Omega}^{q-1}(X) .
$$

One of the interesting questions about $H_{2}^{q}(X)$ is whether or not the map

$$
i: \tilde{\mathfrak{B}}^{2}\left(\bigwedge^{q} X, g\right) \rightarrow H_{2}^{q}(X)
$$


is an isomorphism. If it is, then one says that the Strong Hodge Theorem holds (see [4 and 5]). It follows from Kodaira's theorem that

$$
H_{2}^{q}(X)=\frac{L_{e}^{2}\left(\bigwedge^{q} X\right) \oplus \tilde{\mathfrak{Z}}^{2}\left(\bigwedge^{q} X, g\right)}{d \mathfrak{2}_{c e}^{q-1}(X)} .
$$

Hence (8.3) is 1-1 if $d \Omega_{c e}^{q-1}(X) \subset L_{e}^{2}\left(\wedge^{q} X\right)$ and is onto if $d \Omega_{c e}^{q-1}(X)$ is closed in $L^{2}\left(\wedge^{q} X, g\right)$.

For the remainder of this section we restrict our attention to the manifolds and metrics considered in the preceding sections. Thus $M$ has finitely many ends and $g$ is equivalent to $g_{1}=e^{2 \rho} h$ with $h$ asymptotically translation invariant. An easy consequence of Theorem (7.4) is

Proposition (8.5). If $g$ is equivalent to a metric that is q-bounded above, then (8.3) is $1-1$.

LemMA (8.6). Suppose $g=e^{2 \rho} h$ is an admissible metric on $M$ and that $\rho$ is bounded below. Then $\mathfrak{2}_{c e}^{q}(M) \subset W_{1,0,-1}^{2}\left(\bigwedge^{q} M, g\right)$.

Proof. Let $\sigma \in \mathfrak{2}_{c e}^{q}(M)$. Then $\left(d+d_{g}^{*}\right) \sigma \in L^{2}\left(\bigwedge^{q+1} M, g\right)$ and so $\Delta_{g} \sigma \in$ $W_{-1,0,1}^{2}\left(\wedge^{q} M, g\right)$. It follows from Proposition (4.4) that $K_{1,2} \Delta_{g} \sigma \in W_{-1,0,0}^{2}\left(\bigwedge^{q} M, h\right)=$ $W_{-1,0}^{2}\left(\wedge^{q} M\right)$. However from the definition of the asymptotically translation invariant operator $A(-1+q+n / 2)$ in Theorem (5.2) we see that

$$
K_{1,2} \Delta_{g} \sigma=A(-1+q+n / 2) K_{-1,2} \sigma .
$$

Thus $A(-1+q+n / 2) K_{-1,2} \sigma$ is in $W_{-1,0}^{2}\left(\wedge^{q} M\right)$. Hence using the a priori inequality (3.7.2) we get

$$
\left\|K_{-1,2} \sigma\right\|_{2,1,0} \leqslant c\left(\left\|A\left(-1+q+\frac{n}{2}\right) K_{-1,2} \sigma\right\|_{2,-1,0}+\left\|K_{-1,2} \sigma\right\|_{2,-1,0}\right) .
$$

Since $\rho$ is bounded below and $\sigma \in L^{2}\left(\bigwedge^{q} M, g\right)$, we have that $\sigma \in W_{0,0,-1}^{2}\left(\bigwedge^{q} M, g\right)$. This means that $K_{-1,2} \sigma \in W_{0,0}^{2}\left(\bigwedge^{q} M, g\right)$ and so in $W_{-1,0}^{2}\left(\bigwedge^{q} M, g\right)$. Thus by (8.6.1) we have that $K_{-1,2} \sigma \in W_{1,0}^{2}\left(\wedge^{q} M, g\right)$ which by Proposition (4.4) means that $\sigma \in$ $W_{1,0,-1}^{2}\left(\bigwedge^{q} M, g\right)$.

THEOREM (8.7). Suppose $g$ is equivalent to an admissible metric, $g_{1}=e^{2 \rho} h$, and that $\rho$ is bounded below. Then (8.3) is 1-1. Moreover, if $\limsup _{z \rightarrow \infty} \rho(\omega, z)=\infty$ on at least one end, then (8.3) is not onto for $q>0$; and so the Strong Hodge Theorem does not hold in this case.

Proof. As usual we only need to prove this for $g_{1}$. Furthermore, we may assume that $\rho$ is a function of just $z$ on $M_{\infty}$ and that $h$ is a product metric on $M_{\infty}$. The first part of the theorem follows trivially from Lemma (8.6) and the fact that $d W_{1,0,-1}^{2}\left(\bigwedge^{q-1} M, g\right) \subset L_{e}^{2}\left(\bigwedge^{q} M\right)$.

For the second part of the theorem, let

$$
K=\operatorname{Ker} d_{g}^{*}: W_{1,0,-1}^{2}\left(\bigwedge^{q-1} M, g\right) \rightarrow L^{2}\left(\bigwedge^{q-2} M, g\right) .
$$


It follows from Theorem (5.11) that $K=d_{g}^{*} \tilde{\tilde{W}}_{2,0,-2}^{2}\left(\bigwedge^{q} M, g\right)+\tilde{\mathscr{S}}_{1,0,-1}^{2}\left(\bigwedge^{q-1} M\right)$ where $\tilde{\mathfrak{F}}_{1,0,-1}^{2}\left(\wedge^{q-1} M\right)$ is the finite dimensional space of closed and coclosed forms in $W_{1,0,-1}^{2}\left(\bigwedge^{q-1} M, g\right)$. Note that since $d_{g}^{*} \tilde{\tilde{W}}_{2,0,-2}^{2}\left(\bigwedge^{q} M, g\right)$ is finite codimensional in $K$ and is the image of a continuous operator, it is closed in $K$ and so also in $W_{1,0,-1}^{2}\left(\bigwedge^{q-1} M, g\right)$. Also note that $\mathfrak{\Omega}_{c e}^{q-1}(M) \subset K$.

Suppose $d: K \rightarrow L_{e}^{2}\left(\wedge^{q} M\right)$ is onto; if it is not then a fortiori $d: \Omega_{c e}^{q-1}(M) \rightarrow$ $L_{e}^{2}\left(\wedge^{q} M\right)$ is not. In this case an equivalent norm on $K$ is

$$
\|\sigma\|_{K}=\|\sigma\|\left(W_{0,0,-1}^{2}\left(\wedge^{q-1} M, g\right)\right)+\|d \sigma\|_{2} .
$$

It is easy to see that we then have the commutative diagram of continuous maps

$$
\begin{aligned}
& \mathfrak{2}_{c e}^{q-1}(M) \stackrel{d}{\rightarrow} L_{e}^{2}\left(\bigwedge^{q} M\right) \\
& \downarrow i \quad \nearrow d \\
& \text { K }
\end{aligned}
$$

where $i$ is the inclusion. If $d \Omega_{c e}^{q-1}=L_{e}^{2}\left(\bigwedge^{q} M\right)$, then $i$ must be Fredholm and so $\Omega_{c e}^{q-1}(M)$ is closed in $K$. Now $C_{0}^{\infty}\left(\bigwedge^{q} M\right)$ is dense in $\tilde{W}_{2,0,-2}^{2}\left(\bigwedge^{q} M, g\right)$ and so $d_{g}^{*} C_{0}^{\infty}\left(\bigwedge^{q} M\right)$ is dense in $d_{g}^{*} \tilde{\tilde{W}}_{2,0,-2}^{2}\left(\bigwedge^{q} M, g\right)$. Since $d_{g}^{*} C_{0}^{\infty}\left(\bigwedge^{q} M\right) \subset \mathcal{2}_{c e}^{q-1}(M)$ and $\mathfrak{2}_{c e}^{q-1}(M)$ is closed in $K$, it follows that $d_{g}^{*} \tilde{W}_{2,0,-2}^{2}\left(\bigwedge^{q} M, g\right) \subset \mathfrak{Q}_{c e}^{q-1}(M)$. In fact, since $\Omega_{c e}^{q-1}(M)$ contains no closed forms and $d K=d \Omega_{c e}^{q-1}(M)$, we have that $d_{g}^{*} \tilde{\tilde{W}}_{2,0,-2}^{2}\left(\bigwedge^{q} M, g\right)=\mathfrak{2}_{c e}^{q-1}(M)$.

However this is impossible if $\limsup _{z \rightarrow \infty} \rho(z)=\infty$ on an end of $M$. To see this note that it follows from (2.3) that there is an increasing sequence $\left\{z_{n}\right\}$ such that $z_{1}>4, z_{n+1}-z_{n} \geqslant 4$, and $\rho(z)>n$ on $\left(z_{n}-1, z_{n}+1\right)$. Now let $\nu \in C^{\infty}\left(\wedge^{q} M\right)$ be such that $\nu$ is translation invariant on $M_{\infty}$ and $\nu(\omega, z) \in \wedge^{q} \partial M_{0}$ for $(\omega, z) \in M_{\infty}$. Let $\phi(z) \in C_{0}^{\infty}(\mathbf{R})$ be such that $\operatorname{supp} \phi \subset[-1,1]$ and $\phi \equiv 1$ on $\left[-\frac{1}{2}, \frac{1}{2}\right]$.

Finally take $f \in C^{\infty}(M)$ to be such that $f(z)=\sum_{n=1}^{\infty} n^{-1} \phi\left(z-z_{n}\right)$ on the end of $M$ on which $\lim \sup _{z \rightarrow \infty} \rho(z)=\infty$ and $f \equiv 0$ elsewhere. If $\tau=e^{(q+2-n / 2) \rho} f \nu$, then a calculation shows that $\tau \in W_{2,0,-2}^{2}\left(\bigwedge^{q} M, g\right)$ but $d_{g}^{*} \tau \notin L^{2}\left(\wedge^{q-1} M, g\right)$.

Definition (8.8). Let $G$ be a compact manifold. A function $\rho: G \times \mathbf{R}^{+} \rightarrow \mathbf{R}$ is slowly oscillating if

$$
\sup _{t \in[z / 2, z]}|\rho(\omega, z)-\rho(\omega, t)|<c \quad \text { for some } c \text { independent of } \omega \text { and } z \text {. }
$$

THEOREM (8.9). Suppose $g$ is equivalent to an admissible metric $g_{1}=e^{2 \rho} h$ and that $\rho$ is bounded above. For $i=1,2,3$ let $G_{i}$ be a, possibly empty, union of components of $\partial M_{0}$ satisfying (i) $G_{i} \cap G_{j}=\varnothing$ for $1 \leqslant i, j \leqslant 3$ and $i \neq j$ and (ii) $G_{1} \cup G_{2} \cup G_{3}=$ $\partial M_{0}$. Suppose that $\rho$ is slowly oscillating on $G_{1} \times \mathbf{R}^{+}$and that $\rho(\omega, z) \geqslant(\varepsilon-1) \ln z$ on $G_{1} \times[1, \infty)$ for some $0<\varepsilon<1$. Also assume that on $G_{2} \times[1, \infty)$ both $g_{1}$ is $q$-bounded above and $z e^{\rho}$ is bounded. Finally assume that $\rho$ is $O(z)$ but not $o(z)$ as $z \rightarrow \infty$ on $G_{3} \times[1, \infty)$. Then $(8.3)$ is onto if and only if $H_{D R}^{q-1}\left(G_{1}\right)=0$.

Proof. Suppose $\sigma=d \Psi$ for some $\Psi \in \tilde{W}_{1,0,-1}^{2}\left(\wedge^{q-1} M, g_{1}\right)$. Let $\left\{\phi_{0}, \phi_{1}, \phi_{2}, \phi_{3}\right\}$ be a partition of unity subordinate to the cover $M_{0} \cup\left(\partial M_{0} \times[0,2)\right), G_{1} \times(1, \infty)$, $G_{2} \times(1, \infty), G_{3} \times(1, \infty)$, with $\phi_{i}$ omitted if $G_{i}$ is empty. Then $\sigma=\sum d\left(\phi_{i} \Psi\right)$ and $\phi_{0} \Psi$ is clearly in $2^{q-1}(M)$. Also if $G_{3}$ is not empty, then it follows from Definition (2.3) and the construction of $\tilde{\tilde{W}}_{1,0,-1}^{2}\left(\wedge^{*} M, g_{1}\right)$ that $\phi_{3} \Psi \in \Omega^{q-1}(M)$. 
If $G_{3}=\partial M_{0}$, then we are done. Therefore assume that $G_{1} \cup G_{2}=\bigcup_{l=1}^{K} \Omega_{l}$ where ( $K \leqslant L$ and $\Omega_{1}, \ldots, \Omega_{L}$ ) are the components of $\partial M_{0}$. Also, without loss of generality, assume that $\rho$ just depends on $z$ and $h$ is a product metric on $\left(G_{1} \cup G_{2}\right) \times \mathbf{R}^{+}$.

Let $\sigma_{\infty}=d\left(\phi_{1} \Psi\right)+d\left(\phi_{2} \Psi\right)$. Using the notation of Theorem (7.9) we have that

$$
\begin{aligned}
\sigma_{\infty}= & \sum_{l=-1}^{K} \sum_{i} f_{l i}(z) d z \wedge \eta_{l i}+\sum_{i} h_{-1 i}(z) \Psi_{-1 i} \\
& +\sum_{i} h_{0 i}(z) \lambda_{0 i}^{-1} \tilde{d} \eta_{0 i}+\sum_{l=1}^{K} \sum_{i} h_{l i}(z) \Psi_{l i} .
\end{aligned}
$$

Since $\sigma_{\infty}$ is exact it follows that $h_{-1 i} \equiv 0 ; h_{0 i}^{\prime}=\lambda_{0 i} f_{0 i}$; and $h_{l i} \equiv 0$ for $l>0$. Thus $\sigma_{\infty}=d \tau$ where

(8.9.2) $\tau=\sum_{i} h_{0 i}(z) \lambda_{0 i}^{-1} \eta_{0 i}+\sum_{i} f_{-1 i}(z) d z \wedge \gamma_{-1 i}+\sum_{l=1}^{K} \sum_{i} \int_{0}^{1} z f_{l i}(z t) d t \eta_{l i}$, with $d \gamma_{-1 i}=\eta_{-1 i}$.

Since $\rho$ is bounded above we get from the fact that $\sigma_{\infty} \in L^{2}\left(\wedge^{q} M, g_{1}\right)$ that

$$
\tilde{\tau}=\sum_{i} h_{0 i}(z) \lambda_{0 i}^{-1} \eta_{0 i}+\sum_{i} f_{-1 i}(z) d z \wedge \gamma_{-1 i} \text { is in } L^{2}\left(\wedge^{q-1} M, g_{1}\right) \text {. }
$$

Furthermore, if $\Omega_{l} \subset G_{2}$, then $\int_{0}^{1} z f_{l i}(z t) d t \eta_{l i}$ also is in $L^{2}\left(\bigwedge^{q-1} M, g_{1}\right)$. This follows from the chain of inequalities

$$
\begin{aligned}
\left\|\int_{0}^{1} z f_{l i}(z t) d t \eta_{l i}\right\| & \left(L^{2}\left(\bigwedge^{q-1} M, g_{1}\right)\right) \\
& =\left(\int_{0}^{\infty} \int_{\Omega_{l}} e^{(n-2 q+2) \rho}\left\|\int_{0}^{1} z f_{l i}(z t) d t \eta_{l i}\right\|_{h}^{2} d \omega d z\right)^{1 / 2} \\
& \leqslant c\left(\int_{0}^{\infty} e^{(n-2 q) \rho}\left|\int_{0}^{1} f_{l i}(z t) d t\right|^{2} d z\right)^{1 / 2} \\
& \leqslant c \int_{0}^{1}\left(\int_{0}^{\infty} e^{(n-2 q) \rho(z)}\left|f_{l i}(z t)\right|^{2} d z\right)^{1 / 2} d t \\
& \leqslant c \int_{0}^{1}\left(\int_{0}^{\infty} e^{(n-2 q) \rho(z t)}\left|f_{l i}(z t)\right|^{2} d z\right)^{1 / 2} d t<\infty
\end{aligned}
$$

where the next to last inequality is Minkowski's and the last is $q$-bounded above. Thus if $H_{D R}^{q-1}\left(G_{1}\right)=0$, we have that $\tau \in L^{2}\left(\bigwedge^{q-1} M, g_{1}\right)$ and so $\sigma_{\infty} \in d \Omega^{q-1}(M)$. But this means that $d \tilde{W}_{1,0,-1}^{2}\left(\bigwedge^{q-1} M, g_{1}\right)=d \Omega^{q-1}(M)$ and hence that (8.3) is onto.

If, however, there is an $\Omega_{l} \subset G_{1}$ and $H_{D R}^{q-1}\left(\Omega_{l}\right) \neq 0$, then we can construct an infinite dimensional space of $f_{l i}$ such that $f_{l i}(z) d z \wedge \eta_{l i} \in L^{2}\left(\wedge^{q} M, g_{1}\right)$, but $\int_{0}^{z} f_{l i}(t) d t+k$ is not in $L^{2}\left(\wedge^{q-1} M, g_{1}\right)$ for any $k$. It follows from Kodaira's theorem that in this case $d \mathfrak{\Omega}^{q-1}(M)$ is not closed and so (8.3) is not onto.

More specifically for $-1 / 2-\varepsilon / 2<\alpha<-1 / 2$ let $f_{\alpha} \in C^{\infty}(M)$ be such that $f_{\alpha} \equiv 0$ on $M-\left(\Omega_{l} \times[1, \infty)\right)$ and $f_{\alpha}(\omega, z)=e^{(q-n / 2) \rho^{\alpha}}$ on $\Omega_{l} \times[2, \infty)$. If $\eta_{l 1}$ is a harmonic representative of a nonzero class in $H_{D R}^{q-1}\left(\Omega_{l}\right)$, then $\sigma_{\alpha}=f_{\alpha} d z \wedge \eta_{l 1}$ is exact and in $L^{2}\left(\wedge^{q} M, g_{1}\right)$. Moreover if $\sigma_{\alpha}=d \tau_{\alpha}$, then on $\Omega_{l} \times[1, \infty)$ we have $\tau_{\alpha}=\left(\int_{2}^{z} f_{\alpha}(t) d t+k\right) \eta_{l 1}$. However $\tau_{\alpha} \notin L^{2}\left(\bigwedge^{q-1} M, g_{1}\right)$ for any value of $k$. 
To see this note that

$$
\begin{aligned}
& \left\|\tau_{\alpha}\right\| L^{2}\left(\wedge^{q-1} M, g_{1}\right) \\
& \qquad c\left(\int_{4}^{\infty} e^{(n-2 q+2) \rho(z)}\left|\int_{2}^{z} e^{(q-n / 2) \rho(t)} t^{\alpha} d t+k\right|^{2} d z\right)^{1 / 2} .
\end{aligned}
$$

First suppose that $\int_{2}^{\infty} e^{(q-n / 2) \rho(t)} t^{\alpha} d t=\infty$. Then there is a $z_{0} \geqslant 4$, depending on $k$, such that for $z \geqslant z_{0}$, we have

$$
\int_{2}^{z} e^{(q-n / 2) \rho(t)} t^{\alpha} d t+k \geqslant \frac{1}{2} \int_{2}^{z} e^{(q-n / 2) \rho(t)} t^{\alpha} d t
$$

Thus in this case the right-hand side of (8.9.5) is

$$
\begin{aligned}
& \geqslant c\left(\int_{z_{0}}^{\infty} e^{(n-2 q+2) \rho(z)}\left|\int_{2}^{z} e^{(q-n / 2) \rho(t)} t^{\alpha} d t\right|^{2} d z\right)^{1 / 2} \\
& \geqslant c\left(\int_{z_{0}}^{\infty} z^{2 \varepsilon-2}\left|\int_{z / 2}^{z} e^{(n / 2-q)(\rho(z)-\rho(t))} t^{\alpha} d t\right|^{2} d z\right)^{1 / 2} .
\end{aligned}
$$

From (8.8.1) we get that this last expression is

$$
\begin{aligned}
& \geqslant c\left(\int_{z_{0}}^{\infty} z^{2 \varepsilon-2}\left|z^{\alpha+1}\right|^{2} d z\right)^{1 / 2} \\
& =c\left(\int_{z_{0}}^{\infty} z^{2 \varepsilon+2 \alpha} d z\right)^{1 / 2} \geqslant c\left(\int_{z_{0}}^{\infty} z^{\varepsilon-1} d z\right)^{1 / 2}=\infty .
\end{aligned}
$$

Finally, suppose that $\int_{2}^{\infty} e^{(q-n / 2) \rho(t)} t^{\alpha} d t<\infty$. Since $\alpha>-1$, this means that $q>n / 2$ and so $\int_{4}^{\infty} e^{(n-2 q+2) \rho(t)} d t=\infty$. Hence the only way the right-hand side of (8.9.5) could be finite is if $k=-\int_{2}^{\infty} e^{(q-n / 2) \rho(t)} t^{\alpha} d t$. In this case we have that the right-hand side of (8.9.5)

$$
\begin{aligned}
& =c\left(\int_{4}^{\infty} e^{(n-2 q+2) \rho(z)}\left|\int_{z}^{\infty} e^{(q-n / 2) \rho(t)} t^{\alpha} d t\right|^{2} d z\right)^{1 / 2} \\
& \geqslant c\left(\int_{4}^{\infty} z^{2 \varepsilon-2}\left|\int_{z}^{2 z} e^{(n / 2-q)(\rho(z)-\rho(t))} t^{\alpha} d t\right|^{2} d z\right)^{1 / 2} .
\end{aligned}
$$

Again by (8.8.1) we get that this last expression is

$$
\geqslant c\left(\int_{4}^{\infty} z^{2 \varepsilon-2}\left|z^{\alpha+1}\right|^{2} d z\right)^{1 / 2}=\infty .
$$

\section{REFERENCES}

1. R. Adams, Sobolev spaces, Academic Press, New York, 1975.

2. M. Atiyah, V. Patodi and I. Singer, Spectral asymmetry and riemannian geometry. I, Math. Proc. Cambridge Philos. Soc. 77 (1975), 43-69.

3. J. Cheeger, On the spectral geometry of spaces with conelike singularities, Proc. Nat. Acad. Sci. U.S.A. 76 (1979), 2103-2106.

4. , On the hodge theory of riemannian pseudomanifolds, Geometry of Laplace Operator, Proc. Sympos. Pure Math., vol. 36, Amer. Math. Soc., Providence, R.I., 1980. 
5. J. Cheeger, M. Goresky and R. MacPherson, $L^{2}$-cohomology and intersection homology of singular algebraic varieties, Seminar on Differential Geometry (S. T. Yau, Ed.), Princeton Univ. Press, Princeton, N.J., 1982.

6. J. Cheeger, Spectral geometry of singular riemannian spaces, J. Differential Geom. 18 (1983), 575-657.

7. J. Dodziuk, $L^{2}$ harmonic forms on rotationally symmetric riemannian manifolds, Proc. Amer. Math. Soc. 77 (1979), 395-400.

8. P. Grisvard, Problems de Dirichlet dans un domain non regulier, C.R. Acad. Sci. Paris 278 (1974), 1615-1617.

9. K. Kodaira, Harmonic fields in riemannian geometry (generalized potential theory), Ann. of Math. (2) 50 (1949), 587-665.

10. R. Lockhart and R. McOwen, Elliptic differential operators on non-compact manifolds, Ann. Sci. Norm. Sup. Pisa 12 (1985), 409-447.

11. W. Müller, Spectral theory for riemannian manifolds with cusps and a related trace formula, Math. Nachr. 3 (1983), 197-288.

12. G. De Rham, Variétés différentiables, Hermann, Paris, 1973.

13. S. Zucker, $L_{2}$ cohomology of warped products and arithmetic groups, Invent. Math. 70 (1982), 169-218.

Department of Mathematics, University of Notre Dame, Notre Dame, Indiana 46556 